\title{
Global Exponential Stability of Weighted Pseudo-Almost Periodic Solutions of Neutral Type High-Order Hopfield Neural Networks with Distributed Delays
}

\author{
Lili Zhao and Yongkun Li \\ Department of Mathematics, Yunnan University, Kunming, Yunnan 650091, China \\ Correspondence should be addressed to Yongkun Li; yklie@ynu.edu.cn
}

Received 13 May 2014; Accepted 27 August 2014; Published 3 December 2014

Academic Editor: Elena Braverman

Copyright ( 2014 L. Zhao and Y. Li. This is an open access article distributed under the Creative Commons Attribution License, which permits unrestricted use, distribution, and reproduction in any medium, provided the original work is properly cited.

Some sufficient conditions are obtained for the existence, uniqueness, and global exponential stability of weighted pseudo-almost periodic solutions to a class of neutral type high-order Hopfield neural networks with distributed delays by employing fixed point theorem and differential inequality techniques. The results of this paper are new and they complement previously known results. Moreover, an example is given to show the effectiveness of the proposed method and results.

\section{Introduction}

Since high-order Hopfield neural networks (HHNNs) have stronger approximation property, faster convergence rate, greater storage capacity, and higher fault tolerance than lower-order Hopfield neural networks, the study of highorder Hopfield neural networks has recently gained a lot of attention and there have been extensive results on the problem of the existence and stability of equilibrium points, periodic solutions, and almost periodic solutions of highorder Hopfield neural networks in the literature. We refer the reader to [1-9] and the references cited therein. Also, since it is natural and important that systems will contain some information about the derivative of the past state to further describe and model the dynamics for such complex neural reactions [10], many authors investigated the dynamical behaviors of neutral type neural networks with delays [1125]. Moreover, it is well known that, compared with periodic effects, almost periodic effects are more frequent, and many phenomena exhibit great regularity with being pseudoalmost periodic which allow complex repetitive phenomena to be represented as an almost periodic process plus an ergodic component. However, to the best of our knowledge, few authors have considered the exponential convergence on the pseudo-almost periodic solution for neutral type neural networks with delays. Motivated by the above, in this paper, we consider the following high-order Hopfield neural networks with neutral distributed delays:

$$
\begin{aligned}
& x_{i}^{\prime}(t) \\
& =-c_{i}(t) x_{i}(t)+\sum_{j=1}^{n} a_{i j}(t) \int_{0}^{\infty} d_{i j}(\theta) f_{j}\left(x_{j}(t-\theta)\right) d \theta \\
& +\sum_{j=1}^{n} \alpha_{i j}(t) \int_{0}^{\infty} \beta_{i j}(\theta) h_{j}\left(x_{j}^{\prime}(t-\theta)\right) d \theta \\
& +\sum_{j=1}^{n} \sum_{l=1}^{n} b_{i j l}(t) \int_{0}^{\infty} k_{i j}(\theta) g_{j}\left(x_{j}(t-\theta)\right) d \theta \\
& \quad \times \int_{0}^{\infty} k_{i l}(\theta) g_{l}\left(x_{l}(t-\theta)\right) d \theta+I_{i}(t), \\
& i=1,2, \ldots, n, \quad t>0,
\end{aligned}
$$

where $n$ corresponds to the number of units in a neural network, $x_{i}(t)$ corresponds to the state vector of the $i$ th unit at the time $t, c_{i}(t)$ represents the rate with which the $i$ th unit will reset its potential to the resting state in isolation when disconnected from the network and external inputs, $a_{i j}(t)$ and $b_{i j l}(t)$ are the first- and second-order connection weights of the neural network, $d_{i j}, k_{i j}$ is the kernel, $I_{i}(t)$ denote the 
external inputs at time $t$, and $f_{j}$ and $g_{j}$ are the activation functions of signal transmission.

The initial conditions of (1) are the form

$$
\begin{array}{rlrl}
x_{i}(s) & =\phi_{i}(s), & & x_{i}^{\prime}(s)=\phi_{i}^{\prime}(s), \\
s & \in(-\infty, 0], & i=1,2, \ldots, n,
\end{array}
$$

where $\phi_{i}(\cdot)$ denotes a differential real-value bounded function defined on $(-\infty, 0]$ and satisfies that $\phi_{i}^{\prime}(\cdot)$ is bounded on $(-\infty, 0)$.

To the best of our knowledge, there is no paper published on the global exponential stability and existence of weighted pseudo-almost periodic solution to system (1). Our main purpose of this paper is, for fixed $\mu \in \mathbb{U}_{\infty}^{\text {Inv }}\left(\mathbb{U}_{\infty}^{\text {Inv }}\right.$ will be defined in Section 2), to study the existence, uniqueness, and globally exponential stability of weighted pseudo-almost periodic solution by employing fixed point theorem and differential inequality techniques.

This paper is organized as follows. In Section 2, we introduce some notations and definitions and state some preliminary results which are needed in later sections. In Section 3, we establish some sufficient conditions for the existence of weighted pseudo-almost periodic solutions of (1). In Section 4, we prove that the weighted pseudo-almost periodic solution is globally exponentially stable. In Section 5, we give an example to illustrate the feasibility of our results obtained in previous sections.

\section{Assumptions and Preliminaries}

Throughout this paper we assume that

$\left(\mathrm{H}_{1}\right) c_{i}(t), a_{i j}(t), \alpha_{i j}(t), b_{i j l}(t)$ are almost periodic functions, and $\inf _{t \in \mathbb{R}} c_{i}(t)>0, i, j, l=1,2, \ldots, n$;

$\left(\mathrm{H}_{2}\right)$ there exist positive constants $\epsilon_{i}>0, \vartheta_{i}>0, \varepsilon_{i}>0$, and $G_{i}>0$ such that $\left|f_{i}(x)-f_{i}(y)\right| \leq \epsilon_{i}|x-y|, \mid g_{i}(x)-$ $g_{i}(y)\left|\leq \varepsilon_{i}\right| x-y|,| h_{i}(x)-h_{i}(y)\left|\leq \vartheta_{i}\right| x-y \mid$, and $\left|g_{i}(x)\right| \leq G_{i}$, for all $x, y \in \mathbb{R}$, and $f_{i}(0)=g_{i}(0)=$ $h_{i}(0)=0, i=1,2, \ldots, n$;

$\left(\mathrm{H}_{3}\right)$ for $i, j \in\{1,2, \ldots, n\}$, the delay kernels $d_{i j}, k_{i j}, \beta_{i j}$ : $[0, \infty) \rightarrow \mathbb{R}$ are continuous and integrable with

$$
\begin{gathered}
0 \leq \int_{0}^{\infty}\left|k_{i j}(s)\right| d s \leq k_{i j}^{M}, \quad 0 \leq \int_{0}^{\infty}\left|d_{i j}(s)\right| d s \leq d_{i j}^{M}, \\
0 \leq \int_{0}^{\infty}\left|\beta_{i j}(s)\right| d s \leq \beta_{i j}^{M}
\end{gathered}
$$

$\left(\mathrm{H}_{4}\right)$ for fixed $\mu \in \mathbb{U}^{\mathrm{Inv}}, I_{i}(i=1,2, \ldots, n)$ is a weighted pseudo-almost periodic function.

Definition 1 (see $[26,27]$ ). Let $u: \mathbb{R} \rightarrow \mathbb{R}^{n}$ be continuous in $t ; u(t)$ is said to be almost periodic on $\mathbb{R}$ if, for any $\varepsilon>0$, the set $T(u, \varepsilon)=\{\delta:|u(t+\delta)-u(t)|<\varepsilon, \forall t \in \mathbb{R}\}$ is relatively dense; that is, for all $\varepsilon>0$, it is possible to find a real number $l=l(\varepsilon)>0$; for any interval with length $l(\varepsilon)$, there exists a number $\delta=\delta(\varepsilon)$ in this interval such that $|u(t+\delta)-u(t)|<\varepsilon$, for all $t \in \mathbb{R}$.
For $z=\left(z_{1}, z_{2}, \ldots, z_{n}\right)^{T} \in \mathbb{R}^{n}$, we define $\|z\|=$ $\max _{1 \leq i \leq n}\left|z_{i}\right|$.

Definition 2 (see $[26,27])$. Let $x \in \mathbb{R}^{n}$ and let $Q(t)$ be an $n \times n$ continuous matrix defined on $\mathbb{R}$. The linear system

$$
x^{\prime}(t)=Q(t) x(t)
$$

is said to admit an exponential dichotomy on $\mathbb{R}^{n}$ if there exist positive constants $k, \alpha$; projection $P$ and the fundamental solution matrix $X(t)$ of (4) satisfy

$$
\begin{gathered}
\left\|X(t) P X^{-1}(s)\right\| \leq k e^{-\alpha(t-s)} \quad(\forall t \geq s), \\
\left\|X(t)(I-P) X^{-1}(s)\right\| \leq k e^{-\alpha(s-t)} \quad(\forall s \geq t) .
\end{gathered}
$$

Lemma 3 (see [26, 27]). Let $x(t)$ be an almost periodic function on $\mathbb{R}$; then $x(t)$ is bounded on $\mathbb{R}$ and $x(t)$ is uniformly continuous in $t \in \mathbb{R}$.

The collection of all almost periodic functions which go from $\mathbb{R}$ to $\mathbb{R}^{n}$ will be denoted by $A P\left(\mathbb{R}, \mathbb{R}^{n}\right)$. $A P\left(\mathbb{R}, \mathbb{R}^{n}\right)$ equipped with the sup-norm is a Banach space.

Let $\mathbb{U}$ denote the collection of functions (weights) $\mu$ : $\mathbb{R} \rightarrow(0,+\infty)$, which are locally integrable over $\mathbb{R}$ such that $\mu>0$ almost everywhere. If $\mu \in \mathbb{U}$ and for $r>0$, we set $Q_{r}:=[-r, r]$ and

$$
\mu\left(Q_{r}\right):=\int_{Q_{r}} \mu(x) d x
$$

Let $\mathbb{U}_{\infty}=\left\{\mu \in \mathbb{U}: \inf _{x \in \mathbb{R}} \mu(x)=\mu_{0}>0\right.$ and $\lim _{r \rightarrow \infty} \mu\left(Q_{r}\right)=$ $\infty\}$.

Definition 4 (see [28]). Fix $\mu \in \mathbb{U}_{\infty}$. A continuous function $f: \mathbb{R} \rightarrow \mathbb{X}$ is called weighted pseudo-almost periodic if it can be written as $f=h+\varphi$, with $h \in A P(\mathbb{R}, \mathbb{X})$ and $\varphi \in$ $P A P_{0}(\mathbb{R}, \mathbb{X}, \mu)$, where the space $P A P_{0}(\mathbb{R}, \mathbb{X}, \mu)$ is defined by

$$
\begin{aligned}
& \operatorname{PAP}_{0}(\mathbb{R}, \mathbb{X}, \mu) \\
& \quad=\left\{g \in B C(\mathbb{R}, \mathbb{X}): \lim _{r \rightarrow \infty} \frac{1}{\mu\left(Q_{r}\right)} \int_{Q_{r}}\|g(t)\| \mu(t) d t=0\right\} .
\end{aligned}
$$

The collection of all weighted pseudo-almost periodic functions $f: \mathbb{R} \rightarrow \mathbb{R}^{n}$ will be denoted by $P A P\left(\mathbb{R}, \mathbb{R}^{n}, \mu\right)$.

Remark 5. If $f, g \in P A P\left(\mathbb{R}, \mathbb{R}^{n}, \mu\right)$, then $f+g, f g \in$ $P A P\left(\mathbb{R}, \mathbb{R}^{n}, \mu\right)$; if $f \in P A P\left(\mathbb{R}, \mathbb{R}^{n}, \mu\right), g \in A P\left(\mathbb{R}, \mathbb{R}^{n}\right)$, then $f g \in P A P\left(\mathbb{R}, \mathbb{R}^{n}, \mu\right)$.

Lemma 6 (see [29]). Fix $\mu \in \mathbb{U}_{\infty}$. Suppose that, for any $s \in \mathbb{R}$,

$$
\varlimsup_{|t| \rightarrow \infty} \frac{\mu(t+s)}{\mu(t)}<\infty .
$$

Then $P A P_{0}(\mathbb{R}, \mathbb{X}, \mu)$ is translation-invariant.

Denote $\mathbb{U}_{\infty}^{\mathrm{Inv}}=\left\{\mu \in \mathbb{U}_{\infty}:\right.$ for all $s \in \mathbb{R}, \varlimsup_{\lim _{|t| \rightarrow \infty}}(\mu(t+$ s) $/ \mu(t))<\infty\}$. 
Remark 7. Fix $\mu \in \mathbb{U}_{\infty}^{\text {Inv }}$. Let

$$
\begin{aligned}
P A P^{1}\left(\mathbb{R}, \mathbb{R}^{n}, \mu\right)=\{f & \left(f_{1}(t), f_{2}(t), \ldots, f_{n}(t)\right)^{T}: \\
& \left.f_{i}, f_{i}^{\prime} \in \operatorname{PAP}(\mathbb{R}, \mathbb{R}, \mu), i=1,2, \ldots, n\right\} .
\end{aligned}
$$

Then $P A P^{1}\left(\mathbb{R}, \mathbb{R}^{n}, \mu\right)$ is a Banach space with the norm defined by $\|f\|_{\infty}^{1}=\max \left\{\|f\|_{\infty},\left\|f^{\prime}\right\|_{\infty}\right\}$, where $\|f\|_{\infty}=\sup _{t \in \mathbb{R}}\|f(t)\|$, $\|f(t)\|=\max _{1 \leq i \leq n}\left|f_{i}(t)\right|$.

\section{Existence of Weighted Pseudo-Almost Periodic Solution}

To obtain the existence of weighted pseudo-almost periodic solution to system (1), we need the following lemmas.

Lemma 8 (see $[26,27]$ ). If the linear system (4) admits an exponential dichotomy, then the almost periodic system

$$
x^{\prime}(t)=Q(t) x(t)+g(t)
$$

has a unique almost periodic solution $x(t)$, and

$$
\begin{aligned}
x(t)= & \int_{-\infty}^{t} X(t) P X^{-1}(s) g(s) d s \\
& -\int_{t}^{+\infty} X(t)(I-P) X^{-1}(s) g(s) d s .
\end{aligned}
$$

Lemma 9 (see $[26,27])$. Let $c_{i}(t)$ be an almost periodic function on $\mathbb{R}^{n}$, and

$$
M\left[c_{i}\right]=\lim _{T \rightarrow+\infty} \frac{1}{T} \int_{t}^{t+T} c_{i}(s) d s>0, \quad i=1,2, \ldots, n .
$$

Then the linear system

$$
x^{\prime}(t)=\operatorname{diag}\left(-c_{1}(t),-c_{2}(t), \ldots,-c_{n}(t)\right) x(t)
$$

admits an exponential dichotomy on $\mathbb{R}^{n}$.

Lemma 10. Fix $\mu \in \mathbb{U}_{\infty}^{\text {Inv }}$. If $f: \mathbb{R} \rightarrow \mathbb{R}$ satisfies the Lipschitz condition, $k:[0,+\infty) \rightarrow \mathbb{R}$ is continuous and integrable, and satisfying $0 \leq \int_{0}^{+\infty}|k(u)| d u \leq h$ (where $h$ is a positive constant) and $\varphi \in P A P(\mathbb{R}, \mathbb{R}, \mu)$, then $\Pi: t \rightarrow$ $\left(\int_{0}^{+\infty} k(u) f(\varphi(t-u)) d u\right)$ belongs to $P A P(\mathbb{R}, \mathbb{R}, \mu)$.

Proof. Since $\varphi \in P A P(\mathbb{R}, \mathbb{R}, \mu)$, there exist $\varphi_{1} \in A P(\mathbb{R}, \mathbb{R})$ and $\varphi_{2} \in P A P_{0}(\mathbb{R}, \mathbb{R}, \mu)$ such that $\varphi=\varphi_{1}+\varphi_{2}$; then

$$
\begin{aligned}
\Pi(t)= & \int_{0}^{+\infty} k(u) f\left(\varphi_{1}(t-u)+\varphi_{2}(t)\right) d u \\
= & \int_{0}^{+\infty} k(u) f\left(\varphi_{1}(t-u)\right) d u \\
& +\int_{0}^{+\infty} k(u)\left[f\left(\varphi_{1}(t-u)+\varphi_{2}(t-u)\right)\right. \\
& \left.-f\left(\varphi_{1}(t-u)\right)\right] d u \\
= & : \Pi_{1}(t)+\Pi_{2}(t) .
\end{aligned}
$$

First, we prove that $\Pi_{1} \in A P(\mathbb{R}, \mathbb{R})$. Since $f$ satisfies the Lipschitz condition, there exists a positive constant $L$, such that $\left|f\left(u_{1}\right)-f\left(u_{2}\right)\right| \leq L\left|u_{1}-u_{2}\right|$ for all $u_{1}, u_{2} \in \mathbb{R}$. For any $\varepsilon>0$, since $\varphi_{1} \in A P(\mathbb{R}, \mathbb{R})$, it is possible to find a real number $l=l(\varepsilon)>0$; for any interval with length $l(\varepsilon)$, there exists a number $\tau=\tau(\varepsilon)$ in this interval such that $\left|\varphi_{1}(u+\tau)-\varphi_{1}(u)\right|<\varepsilon / L k$ for all $t \in \mathbb{R}$; then

$$
\begin{aligned}
& \left|\Pi_{1}(t+\tau)-\Pi_{1}(t)\right| \\
& =\mid \int_{0}^{+\infty} k(u) f\left(\varphi_{1}(t+\tau-u)\right) d u \\
& \quad \quad-\int_{0}^{+\infty} k(u) f\left(\varphi_{1}(t-u)\right) d u \mid \\
& =\left|\int_{0}^{+\infty} k(u)\left[f\left(\varphi_{1}(t+\tau-u)\right)-f\left(\varphi_{1}(t-u)\right)\right] d u\right| \\
& =\left|\int_{-\infty}^{t} k(t-u)\left[f\left(\varphi_{1}(u+\tau)\right)-f\left(\varphi_{1}(u)\right)\right] d u\right| \\
& \leq\left|\int_{-\infty}^{t}\right| k(u)|L| \varphi_{1}(u+\tau)-\varphi_{1}(u) \mid d u<k L \frac{\varepsilon}{k L}=\varepsilon
\end{aligned}
$$

which implies that $\Pi_{1} \in A P(\mathbb{R}, \mathbb{R})$. Next, we prove that $\Pi_{2} \epsilon$ $P A P_{0}(\mathbb{R}, \mathbb{R}, \mu)$. Consider

$$
\begin{aligned}
& \lim _{r \rightarrow \infty} \frac{1}{\mu\left(Q_{r}\right)} \int_{Q_{r}}\left|\Pi_{2}(t)\right| d t \\
& =\lim _{r \rightarrow \infty} \int_{Q_{r}} \mid \int_{0}^{+\infty} k(u)\left[f\left(\varphi_{1}(t-u)+\varphi_{2}(t-u)\right)\right. \\
& \left.-f\left(\varphi_{1}(t-u)\right)\right] d u \mid \mu(t) d t \\
& \leq \lim _{r \rightarrow \infty} \frac{L}{\mu\left(Q_{r}\right)} \int_{0}^{+\infty}|k(u)|\left(\int_{Q_{r}}\left|\varphi_{2}(t-u)\right| \mu(t) d t\right) d u .
\end{aligned}
$$

Consider the following function:

$$
\Gamma_{r}(u)=\frac{1}{\mu\left(Q_{r}\right)} \int_{Q_{r}}\left|\varphi_{2}(t-u)\right| \mu(t) d t
$$

obviously, $\Gamma_{r}(u)$ is bounded, and, by using Lemma 6, we have $\lim _{r \rightarrow \infty} \Gamma_{r}(u)=0$. Consequently, by the Lebesgue dominated convergence theorem, we get

$$
\begin{aligned}
& \lim _{r \rightarrow \infty} \frac{1}{\mu\left(Q_{r}\right)} \int_{Q_{r}}\left|\Pi_{2}(t)\right| d t \\
& \quad \leq L \lim _{r \rightarrow \infty} \int_{0}^{+\infty}|k(u)| \Gamma_{r}(u) d u=0,
\end{aligned}
$$

which implies that $\Pi_{2} \in \operatorname{PAP}_{0}(\mathbb{R}, \mathbb{R}, \mu)$. The proof is complete. 
Lemma 11. Fix $\mu \in \mathbb{U}_{\infty}^{I n v}$. Suppose that assumptions $\left(H_{1}\right)-\left(H_{3}\right)$ hold. For each $\varphi=\left(\varphi_{1}, \varphi_{2}, \ldots, \varphi_{n}(t)\right)^{T} \in \operatorname{PAP}^{1}\left(\mathbb{R}, \mathbb{R}^{n}, \mu\right)$, define a nonlinear operator as follows:

$T_{\varphi}(t)$

$$
\begin{aligned}
= & \left(x_{\varphi_{1}}(t), x_{\varphi_{2}}(t), \ldots, x_{\varphi_{n}}(t)\right)^{T} \\
= & \left(\int_{-\infty}^{t} e^{-\int_{s}^{t} c_{1}(u) d u} \Gamma_{1}(s) d s,\right. \\
& \left.\quad \int_{-\infty}^{t} e^{-\int_{s}^{t} c_{2}(u) d u} \Gamma_{2}(s) d s, \ldots, \int_{-\infty}^{t} e^{-\int_{s}^{t} c_{n}(u) d u} \Gamma_{n}(s) d s\right)^{T},
\end{aligned}
$$

where, for $i=1,2, \ldots, n$,

$$
\begin{aligned}
\Gamma_{i}(s)= & \sum_{j=1}^{n} a_{i j}(s) \int_{0}^{+\infty} d_{i j}(\theta) f_{j}\left(\varphi_{j}(s-\theta)\right) d \theta \\
& +\sum_{j=1}^{n} \alpha_{i j}(s) \int_{0}^{+\infty} \beta_{i j}(\theta) h_{j}\left(\varphi_{j}^{\prime}(s-\theta)\right) d \theta \\
& +\sum_{j=1}^{n} \sum_{l=1} b_{i j l}(s) \int_{0}^{\infty} k_{i j}(\theta) g_{j}\left(\varphi_{j}(s-\theta)\right) d \theta \\
& \times \int_{0}^{\infty} k_{i l}(\theta) g_{l}\left(\varphi_{l}(s-\theta)\right) d \theta+I_{i}(s),
\end{aligned}
$$

and then $T$ maps $P A P^{1}\left(\mathbb{R}, \mathbb{R}^{n}, \mu\right)$ into itself.

Proof. Let $\varphi \in P A P^{1}\left(\mathbb{R}, \mathbb{R}^{n}, \mu\right)$; by Lemma 10 and in view of $\left(\mathrm{H}_{1}\right)-\left(\mathrm{H}_{4}\right)$, we have $\Gamma_{i} \in P A P(\mathbb{R}, \mathbb{R}, \mu)(i=1,2, \ldots, n)$; that is, $\Gamma_{i}$ can be rewritten as $\Gamma_{i}=\Gamma_{i 1}+\Gamma_{i 2}$, where $\Gamma_{i 1} \in A P(\mathbb{R}, \mathbb{R})$ and $\Gamma_{i 2} \in P A P_{0}(\mathbb{R}, \mathbb{R}, \mu)$. Hence,

$$
\begin{aligned}
x_{\varphi_{i}}(t) & =\int_{-\infty}^{t} e^{-\int_{s}^{t} c_{i}(u) d u} \Gamma_{i}(s) d s \\
& =\int_{-\infty}^{t} e^{-\int_{s}^{t} c_{i}(u) d u} \Gamma_{i 1}(s) d s+\int_{-\infty}^{t} e^{-\int_{s}^{t} c_{i}(u) d u} \Gamma_{i 2}(s) d s \\
& =: T_{i 1}(t)+T_{i 2}(t), \quad i=1,2, \ldots, n .
\end{aligned}
$$

Consider the following almost periodic system:

$$
x_{i}^{\prime}(t)=-c_{i}(t) x_{i}(t)+\Gamma_{i 1}(t), \quad i=1,2, \ldots, n .
$$

Since $M\left[c_{i}\right]>0(i=1,2, \ldots, n)$, from Lemmas 8 and 9 , system (22) has an almost periodic solution which can be expressed as follows:

$$
\begin{aligned}
& z(t) \\
& =\left(\int_{-\infty}^{t} e^{-\int_{s}^{t} c_{1}(u) d u} \Gamma_{11}(s) d s,\right. \\
& \left.\quad \int_{-\infty}^{t} e^{-\int_{s}^{t} c_{2}(u) d u} \Gamma_{21}(s) d s, \ldots, \int_{-\infty}^{t} e^{-\int_{s}^{t} c_{n}(u) d u} \Gamma_{n 1}(s) d s\right)^{T} ;
\end{aligned}
$$

that is, $T_{i 1} \in A P(\mathbb{R}, \mathbb{R})(i=1,2, \ldots, n)$. Let

$$
J_{i}=\frac{1}{\mu\left(Q_{r}\right)} \int_{Q_{r}}\left|\int_{-\infty}^{t} e^{-\int_{s}^{t} c_{i}(u) d u} \Gamma_{i 2}(s) d s\right| \mu(t) d t
$$

in order to prove that $T_{i 2} \in P A P_{0}(\mathbb{R}, \mathbb{R}, \mu)$, we will prove $\lim _{r \rightarrow \infty} J_{i}=0(i=1,2, \ldots, n)$. Notice that

$$
\begin{aligned}
J_{i} & =\frac{1}{\mu\left(Q_{r}\right)} \int_{Q_{r}}\left|\int_{-\infty}^{t} e^{-\int_{s}^{t} c_{i}(u) d u} \Gamma_{i 2}(s) d s\right| \mu(t) d t \\
& \leq \frac{1}{\mu\left(Q_{r}\right)} \int_{Q_{r}}\left|\int_{-\infty}^{t} e^{-c_{i}^{m}(t-s)} \Gamma_{i 2}(s) d s\right| \mu(t) d t \\
& =\frac{1}{\mu\left(Q_{r}\right)} \int_{Q_{r}}\left|\int_{0}^{+\infty} e^{-c_{i}^{m} s} \Gamma_{i 2}(t-s) d s\right| \mu(t) d t \\
& \leq \frac{1}{\mu\left(Q_{r}\right)} \int_{Q_{r}}\left(\int_{0}^{+\infty} e^{-c_{i}^{m} s}\left|\Gamma_{i 2}(t-s)\right| d s\right) \mu(t) d t \\
& =\int_{0}^{+\infty} e^{-c_{i}^{m} s}\left(\frac{1}{\mu\left(Q_{r}\right)} \int_{Q_{r}}\left|\Gamma_{i 2}(t-s)\right| \mu(t) d t\right) d s
\end{aligned}
$$

where $c_{i}^{m}=\inf _{t \in \mathbb{R}} c_{i}(t)(i=1,2, \ldots, n)$. Since the function $\Gamma_{i 2} \in P A P_{0}(\mathbb{R}, \mathbb{R}, \mu)$, then the functions $\Pi_{i}(s)$ defined by

$$
\Pi_{i}(s)=\frac{1}{\mu\left(Q_{r}\right)} \int_{Q_{r}}\left|\Gamma_{i 2}(t-s)\right| \mu(t) d t
$$

are bounded and satisfy $\lim _{r \rightarrow \infty} \Pi_{i}(s)=0$. Consequently, by the Lebesgue dominated convergence theorem, we have

$$
\lim _{r \rightarrow \infty} J_{i}=0, \quad i=1,2, \ldots, n
$$

that is, $T_{i 2} \in P A P_{0}(\mathbb{R}, \mathbb{R}, \mu)(i=1,2, \ldots, n)$. Now, we can get that $x_{\varphi_{i}} \in P A P(\mathbb{R}, \mathbb{R}, \mu)$, and

$$
x_{\varphi_{i}}^{\prime}(t)=-c_{i}(t) x_{\varphi_{i}}(t)+\Gamma_{i}(t)
$$

obviously, $x_{\varphi_{i}}^{\prime}(t) \in P A P(\mathbb{R}, \mathbb{R}, \mu)$; that is, $T$ maps $P A P^{1}\left(\mathbb{R}, \mathbb{R}^{n}, \mu\right)$ into itself.

For the sake of convenience, we introduce the following notations:

$$
\begin{array}{cc}
c_{i}^{m}=\inf _{t \in \mathbb{R}} c_{i}(t), & c_{i}^{M}=\sup _{t \in \mathbb{R}} c_{i}(t), \\
\overline{I_{i}}=\sup _{t \in \mathbb{R}}\left|I_{i}(t)\right|, & \overline{a_{i j}}=\sup _{t \in \mathbb{R}}\left|a_{i j}(t)\right|, \\
\overline{\alpha_{i j}}=\sup _{t \in \mathbb{R}}\left|\alpha_{i j}(t)\right|, & \overline{b_{i j l}}=\sup _{t \in \mathbb{R}}\left|b_{i j l}(t)\right|, \\
& i, j, l=1,2, \ldots, n,
\end{array}
$$




$$
\begin{gathered}
A=\max \left\{\max _{1 \leq i \leq n}\left\{\frac{\overline{I_{i}}}{c_{i}^{m}}\right\}, \max _{1 \leq i \leq n}\left\{\overline{I_{i}}+c_{i}^{M} \frac{\overline{I_{i}}}{c_{i}^{m}}\right\}\right\}, \\
B=\max _{1 \leq i \leq n}\left\{\max \left\{\frac{1}{c_{i}^{m}}, 1+\frac{c_{i}^{M}}{c_{i}^{m}}\right\}\right. \\
\times \sum_{j=1}^{n}\left[\overline{a_{i j}} d_{i j}^{M}\left|f_{j}(0)\right|+\overline{\alpha_{i j}} \beta_{i j}^{M}\left|h_{j}(0)\right|\right. \\
\left.\left.+\sum_{l=1}^{n} \overline{b_{i j l}} G_{l} G_{j} k_{i j}^{M} k_{i l}^{M}\right]\right\}, \\
\phi_{0}(t) \quad\left(\int_{-\infty}^{t} e^{-\int_{s}^{t} c_{1}(u) d u} I_{1}(s) d s,\right. \\
\left.\int_{-\infty}^{t} e^{-\int_{s}^{t} c_{2}(u) d u} I_{2}(s) d s, \ldots, \int_{-\infty}^{t} e^{-\int_{s}^{t} c_{n}(u) d u} I_{n}(s) d s\right)^{T} .
\end{gathered}
$$

Theorem 12. Suppose that $\left(H_{1}\right)-\left(H_{4}\right)$ and

$$
\begin{aligned}
=\max _{1 \leq i \leq n}\{ & \max \left\{\frac{1}{c_{i}^{m}}, 1+\frac{c_{i}^{M}}{c_{i}^{m}}\right\} \\
& \left.\times \sum_{j=1}^{n}\left[\overline{a_{i j}} d_{i j}^{M} \epsilon_{j}+\overline{\alpha_{i j}} \beta_{i j}^{M} \vartheta_{j}+2 \varepsilon_{j} \sum_{l=1}^{n} \overline{b_{i j l}} G_{l} k_{i j}^{M} k_{i l}^{M}\right]\right\}
\end{aligned}
$$$$
\xi
$$$$
<1
$$

hold; then there exists a unique continuously differentiable weighted pseudo-almost periodic solution of system (1) in the region $E=\left\{\phi \mid \phi \in P A P^{1}\left(\mathbb{R}, \mathbb{R}^{n}, \mu\right),\left\|\phi-\phi_{0}\right\|_{\infty}^{1} \leq(A \xi+\right.$ $B) /(1-\xi)\}$.

Proof. For all $\phi \in P A P^{1}\left(\mathbb{R}, \mathbb{R}^{n}, \mu\right)$, from Lemma 11, T maps $P A P^{1}\left(\mathbb{R}, \mathbb{R}^{n}, \mu\right)$ into itself. By the definition of the norm of Banach space $P A P^{1}\left(\mathbb{R}, \mathbb{R}^{n}, \mu\right)$, we have

$$
\begin{aligned}
& \left\|\phi_{0}\right\|_{\infty}^{1} \\
& =\max \left\{\left\|\phi_{0}\right\|_{\infty},\left\|\phi_{0}^{\prime}\right\|_{\infty}\right\} \\
& =\max \left\{\sup _{t \in \mathbb{R}} \max _{1 \leq i \leq n}\left|\int_{-\infty}^{t} I_{i}(s) e^{-\int_{s}^{t} c_{i}(u) d u} d s\right|,\right. \\
& \left.\quad \sup _{t \in \mathbb{R}} \max _{1 \leq i \leq n}\left|I_{i}(t)-\int_{-\infty}^{t} I_{i}(s) c_{i}(t) e^{-\int_{s}^{t} c_{i}(u) d u} d s\right|\right\} \\
& \leq \max \left\{\max _{1 \leq i \leq n}\left\{\frac{\overline{I_{i}}}{c_{i}^{m}}\right\}, \max _{1 \leq i \leq n}\left\{\bar{I}_{i}+c_{i}^{M} \frac{\overline{I_{i}}}{c_{i}^{m}}\right\}\right\}=A .
\end{aligned}
$$

Hence, for all $\phi \in E=\left\{\phi \mid \phi \in P A P^{1}(\mathbb{R}, \mathbb{R}, \mu),\left\|\phi-\phi_{0}\right\|_{\infty}^{1} \leq\right.$ $(A \xi+B) /(1-\xi)\}$, we obtain

$$
\left\|\phi_{0}\right\|_{\infty}^{1} \leq\left\|\phi-\phi_{0}\right\|_{\infty}^{1}+\left\|\phi_{0}\right\|_{\infty}^{1} \leq \frac{A \xi+B}{1-\xi}+A=\frac{A+B}{1-\xi}
$$

Next, we show that $T$ maps the closed set $E$ into itself. In fact, for any $\phi \in E$, we obtain by $\left(\mathrm{H}_{2}\right)-\left(\mathrm{H}_{3}\right)$ that

$$
\begin{gathered}
\left\|T \phi-\phi_{0}\right\|_{\infty} \\
=\sup _{t \in \mathbb{R}} \max _{1 \leq i \leq n}\left\{\mid \int_{-\infty}^{t} e^{-\int_{s}^{t} c_{i}(u) d u}\right. \\
\times\left[\sum_{j=1}^{n} a_{i j}(s) \int_{0}^{\infty} d_{i j}(\theta) f_{j}\left(\phi_{j}(s-\theta)\right) d \theta\right. \\
+\sum_{j=1}^{n} \alpha_{i j}(s) \int_{0}^{\infty} \beta_{i j}(\theta) h_{j}\left(\phi_{j}^{\prime}(s-\theta)\right) d \theta \\
+\sum_{j=1}^{n} \sum_{l=1}^{n} b_{i j l}(s) \int_{0}^{\infty} k_{i j}(\theta) g_{j} \\
\left.\times \int_{0}^{\infty} k_{i l}(\theta) g_{l}(s-\theta)\right) d \theta \\
\left.\left.\times\left(\phi_{l}(s-\theta)\right) d \theta\right] d s \mid\right\}
\end{gathered}
$$

$$
\begin{gathered}
\leq \sup _{t \in \mathbb{R}} \max _{1 \leq i \leq n}\left\{\int_{-\infty}^{t} e^{-\int_{s}^{t} c_{i}(u) d u}\right. \\
\times\left[\sum _ { j = 1 } ^ { n } \left(\left|a_{i j}(s)\right| \int_{0}^{\infty}\left|d_{i j}(\theta)\right|\right.\right. \\
\times\left[\mid f_{j}\left(\phi_{j}(s-\theta)\right)\right. \\
-f_{j}(0) \mid \\
\left.\left.+\left|f_{j}(0)\right|\right] d \theta\right) \\
+\sum_{j=1}^{n}\left|\alpha_{i j}(s)\right| \begin{array}{r}
\left|\beta_{i j}(\theta)\right| \\
\times\left[\left|h_{j}\left(\phi_{j}^{\prime}(s-\theta)\right)-h_{j}(0)\right|\right. \\
\left.+\left|h_{j}(0)\right|\right] d \theta
\end{array}
\end{gathered}
$$




$$
\begin{aligned}
& +\sum_{j=1}^{n} \sum_{l=1}^{n}\left|b_{i j l}(s)\right| \\
& \times\left[\int_{0}^{\infty}\left|k_{i j}(\theta)\right|\right. \\
& \times\left|g_{j}\left(\phi_{j}(s-\theta)\right)-g_{j}(0)\right| d \theta \\
& \times \int_{0}^{\infty}\left|k_{i l}(\theta)\right| \\
& \left.\times\left|g_{l}\left(\phi_{l}(s-\theta)\right)\right| d \theta\right] \\
& +\sum_{j=1}^{n} \sum_{l=1}^{n}\left|b_{i j l}(s)\right| \int_{0}^{\infty}\left|k_{i j}(\theta)\right|\left|g_{j}(0)\right| d \theta \\
& \times \int_{0}^{\infty}\left|k_{i l}(\theta)\right| \\
& \times\left|g_{l}\left(\phi_{l}(s-\theta)\right)-g_{l}(0)\right| d \theta \\
& +\sum_{j=1}^{n} \sum_{l=1}^{n}\left|b_{i j l}(s)\right| \int_{0}^{\infty}\left|k_{i j}(\theta)\right|\left|g_{j}(0)\right| d \theta \\
& \left.\left.\times \int_{0}^{\infty}\left|k_{i l}(\theta)\right|\left|g_{l}(0)\right| d \theta\right] d s\right\} \\
& \leq \sup _{t \in \mathbb{R}} \max _{1 \leq i \leq n}\left\{\int_{-\infty}^{t} e^{-\int_{s}^{t} c_{i}(u) d u}\right. \\
& \times\left[\sum_{j=1}^{n} \overline{a_{i j}} \int_{0}^{\infty}\left|d_{i j}(\theta)\right|\right. \\
& \times\left(\epsilon_{j}\left|\phi_{j}(s-\theta)\right|+\left|f_{j}(0)\right|\right) d \theta \\
& +\sum_{j=1}^{n} \overline{\alpha_{i j}} \int_{0}^{\infty}\left|\beta_{i j}(\theta)\right| \\
& \times\left(\vartheta_{j}\left|\phi_{j}^{\prime}(s-\theta)\right|+\left|h_{j}(0)\right|\right) d \theta \\
& +\sum_{j=1}^{n} \sum_{l=1}^{n} \overline{b_{i j l}} G_{l} \varepsilon_{j} k_{i l}^{M} \int_{0}^{\infty}\left|k_{i j}(\theta)\right| \\
& \times\left|\phi_{j}(s-\theta)\right| d \theta \\
& +\sum_{j=1}^{n} \sum_{l=1}^{n} \overline{b_{i j l}} G_{j} \varepsilon_{l} k_{i j}^{M} \int_{0}^{\infty}\left|k_{i l}(\theta)\right| \\
& \times\left|\phi_{l}(s-\theta)\right| d \theta \\
& \left.\left.+\sum_{j=1}^{n} \sum_{l=1}^{n} \bar{b}_{i j l} G_{j} G_{l} k_{i j}^{M} k_{i l}^{M}\right] d s\right\} \\
& =\max _{1 \leq i \leq n}\left\{\frac{1}{c_{i}^{m}}\right. \\
& \times\left[\sum _ { j = 1 } ^ { n } \left(\overline{a_{i j}} d_{i j}^{M} \epsilon_{j}+\overline{\alpha_{i j}} \beta_{i j}^{M} \vartheta_{j}\right.\right. \\
& \left.+2 \sum_{l=1}^{n} \overline{b_{i j l}} G_{l} \varepsilon_{j} k_{i j}^{M} k_{i l}^{M}\right)\|\phi\|_{\infty}^{1} \\
& +\sum_{j=1}^{n}\left(\overline{a_{i j}} d_{i j}^{M}\left|f_{j}(0)\right|+\overline{\alpha_{i j}} \beta_{i j}^{M}\left|h_{j}(0)\right|\right. \\
& \left.\left.\left.+\sum_{l=1}^{n} \overline{b_{i j l}} G_{j} G_{l} k_{i j}^{M} k_{i l}^{M}\right)\right] d s\right\} \\
& \times\left[\sum _ { j = 1 } ^ { n } \left(\overline{a_{i j}} d_{i j}^{M} \epsilon_{j}+\overline{\alpha_{i j}} \beta_{i j}^{M} \vartheta_{j}\right.\right. \\
& \left.+2 \sum_{l=1}^{n} \overline{b_{i j l}} G_{l} \varepsilon_{j} k_{i j}^{M} k_{i l}^{M}\right)\|\phi\|_{\infty}^{1} \\
& +\sum_{j=1}^{n}\left(\overline{a_{i j}} d_{i j}^{M}\left|f_{j}(0)\right|+\overline{\alpha_{i j}} \beta_{i j}^{M}\left|h_{j}(0)\right|\right. \\
& \left.\left.\left.+\sum_{l=1}^{n} \overline{b_{i j l}} G_{j} G_{l} k_{i j}^{M} k_{i l}^{M}\right)\right]\right\} \text {. } \\
& \left\|\left(T \phi-\phi_{0}\right)^{\prime}\right\|_{\infty} \\
& =\sup _{t \in \mathbb{R}} \max _{1 \leq i \leq n}\left\{\mid \sum_{j=1}^{n} a_{i j}(t)\right.
\end{aligned}
$$




$$
\begin{aligned}
& \times \int_{0}^{\infty} d_{i j}(\theta) f_{j}\left(\phi_{j}(t-\theta)\right) d \theta \\
& +\sum_{j=1}^{n} \alpha_{i j}(t) \int_{0}^{\infty} \beta_{i j}(\theta) h_{j}\left(\phi_{j}^{\prime}(t-\theta)\right) d \theta \\
& +\sum_{j=1}^{n} \sum_{l=1}^{n} b_{i j l}(t) \int_{0}^{\infty} k_{i j}(\theta) g_{j}\left(\phi_{j}(t-\theta)\right) d \theta \\
& \times \int_{0}^{\infty} k_{i l}(\theta) g_{l}\left(\phi_{l}(t-\theta)\right) d \theta \\
& -\int_{-\infty}^{t} c_{i}(t) e^{-\int_{s}^{t} c_{i}(u) d u} \\
& \times\left[\sum_{j=1}^{n} a_{i j}(s) \int_{0}^{\infty} d_{i j}(\theta) f_{j}\left(\phi_{j}(s-\theta)\right) d \theta\right. \\
& +\sum_{j=1}^{n} \alpha_{i j}(s) \int_{0}^{\infty} \beta_{i j}(\theta) h_{j}\left(\phi_{j}^{\prime}(s-\theta)\right) d \theta \\
& +\sum_{j=1}^{n} \sum_{l=1}^{n} b_{i j l}(s) \\
& \times \int_{0}^{\infty} k_{i j}(\theta) g_{j}\left(\phi_{j}(s-\theta)\right) d \theta \\
& \times \int_{0}^{\infty} k_{i l}(\theta) g_{l} \\
& \left.\left.\times\left(\phi_{l}(s-\theta)\right) d \theta\right] d s \mid\right\} \\
& \leq \sup _{t \in \mathbb{R}} \max _{1 \leq i \leq n}\left\{\sum _ { j = 1 } ^ { n } \left(\overline{a_{i j}} d_{i j}^{M} \epsilon_{j}+\overline{\alpha_{i j}} \beta_{i j}^{M} \vartheta_{j}\right.\right. \\
& \left.+2 \varepsilon_{j} \sum_{l=1}^{n} \overline{b_{i j l}} G_{l} k_{i j}^{M} k_{i l}^{M}\right)\|\phi\|_{\infty}^{1} \\
& +\sum_{j=1}^{n}\left(\overline{a_{i j}} d_{i j}^{M}\left|f_{j}(0)\right|+\overline{\alpha_{i j}} \beta_{i j}^{M}\left|h_{j}(0)\right|\right. \\
& \left.+G_{j} \sum_{l=1}^{n} \overline{b_{i j l}} G_{l} k_{i j}^{M} k_{i l}^{M}\right) \\
& \begin{array}{l}
+\int_{-\infty}^{t} c_{i}^{M} e^{-c_{i}^{m}(t-s)} \\
\quad \times\left[\sum _ { j = 1 } ^ { n } \left(\overline{a_{i j}} d_{i j}^{M} \epsilon_{j}+\overline{\alpha_{i j}} \beta_{i j}^{M} \vartheta_{j}\right.\right.
\end{array} \\
& \left.+2 \varepsilon_{j} \sum_{l=1}^{n} \overline{b_{i j l}} G_{l} k_{i j}^{M} k_{i l}^{M}\right)\|\phi\|_{\infty}^{1}
\end{aligned}
$$$$
\begin{array}{r}
+\sum_{j=1}^{n}\left(\overline{a_{i j}} d_{i j}^{M}\left|f_{j}(0)\right|+\overline{\alpha_{i j}} \beta_{i j}^{M}\left|h_{j}(0)\right|\right. \\
\left.\left.\left.+G_{j} \sum_{l=1}^{n} \overline{b_{i j l}} G_{l} k_{i j}^{M} k_{i l}^{M}\right)\right] d s\right\}
\end{array}
$$$$
\leq \max _{1 \leq i \leq n}\left\{( 1 + \frac { c _ { i } ^ { M } } { c _ { i } ^ { m } } ) \left[\sum _ { j = 1 } ^ { n } \left(\overline{a_{i j}} d_{i j}^{M} \epsilon_{j}+\overline{\alpha_{i j}} \beta_{i j}^{M} \vartheta_{j}\right.\right.\right.
$$$$
\left.+2 \varepsilon_{j} \sum_{l=1}^{n} \overline{b_{i j l}} G_{l} k_{i j}^{M} k_{i l}^{M}\right)\|\phi\|_{\infty}^{1}
$$$$
+\sum_{j=1}^{n}\left(\overline{a_{i j}} d_{i j}^{M}\left|f_{j}(0)\right|+\overline{\alpha_{i j}} \beta_{i j}^{M}\left|h_{j}(0)\right|\right.
$$$$
\left.\left.\left.+G_{j} \sum_{l=1}^{n} \overline{b_{i j l}} G_{l} k_{i j}^{M} k_{i l}^{M}\right)\right]\right\} .
$$

Thus, it follows from (33) and (34) that

$$
\begin{aligned}
& \left\|T \phi-\phi_{0}\right\|_{\infty}^{1} \\
& \leq \max \left\{\frac{1}{c_{i}^{m}}, 1+\frac{c_{i}^{M}}{c_{i}^{m}}\right\} \\
& \times\left[\sum_{j=1}^{n}\left(\overline{a_{i j}} d_{i j}^{M} \epsilon_{j}+\overline{\alpha_{i j}} \beta_{i j}^{M} \vartheta_{j}+2 \varepsilon_{j} \sum_{l=1}^{n} \overline{b_{i j l}} G_{l} k_{i j}^{M} k_{i l}^{M}\right)\right. \\
& \left.\quad \times\|\phi\|_{\infty}^{1}\right]+B \\
& \leq \xi\|\phi\|_{\infty}^{1}+B \leq \frac{A \xi+B}{1-\xi}
\end{aligned}
$$

which implies that $T \phi \in E$. So, the mapping $T$ is a selfmapping from $E$ to $E$. Finally, we prove that $T$ is a contraction mapping of the $E$. In fact, in view of $\left(\mathrm{H}_{2}\right)$, for any $\phi, \psi \in E$, we obtain

$$
\begin{aligned}
& \|T \phi-T \psi\|_{\infty} \\
& =\sup _{t \in \mathbb{R}} \max _{1 \leq i \leq n}\left\{\mid \int_{-\infty}^{t} e^{-\int_{s}^{t} c_{i}(u) d u}\right. \\
& \times\left[\sum_{j=1}^{n} a_{i j}(s)\right.
\end{aligned}
$$


8

Abstract and Applied Analysis

$$
\begin{aligned}
& \times \int_{0}^{\infty} d_{i j}(\theta)\left[f_{j}\left(\phi_{j}(s-\theta)\right)\right. \\
& \left.-f_{j}\left(\psi_{j}(s-\theta)\right)\right] d \theta \\
& +\sum_{j=1}^{n} \alpha_{i j}(s) \\
& \leq \max _{1 \leq i \leq n}\left\{\frac{1}{c_{i}^{m}} \sum_{j=1}^{n}\left[\overline{a_{i j}} d_{i j}^{M} \epsilon_{j}+\overline{\alpha_{i j}} \beta_{i j}^{M} \vartheta_{j}+2 \sum_{l=1}^{n} \overline{b_{i j l}} G_{l} \varepsilon_{j} k_{i j}^{M} k_{i l}^{M}\right]\right\} \\
& \times\|\phi-\psi\|_{\infty}^{1} \leq \xi\|\phi-\psi\|_{\infty}^{1}, \\
& \left\|(T \phi-T \psi)^{\prime}\right\|_{\infty} \\
& \times \int_{0}^{\infty} \beta_{i j}(\theta)\left[h_{j}\left(\phi_{j}^{\prime}(s-\theta)\right)\right. \\
& =\sup _{t \in \mathbb{R}} \max _{1 \leq i \leq n}\left\{\mid \sum_{j=1}^{n} a_{i j}(t)\right. \\
& \left.-h_{j}\left(\psi_{j}^{\prime}(s-\theta)\right)\right] d \theta \\
& +\sum_{j=1}^{n} \sum_{l=1}^{n} b_{i j l}(s) \\
& \times \int_{0}^{\infty} d_{i j}(\theta) \\
& \times\left[f_{j}\left(\phi_{j}(t-\theta)\right)-f_{j}\left(\psi_{j}(t-\theta)\right)\right] d \theta \\
& \times \int_{0}^{\infty} k_{i j}(\theta)\left[g_{j}\left(\phi_{j}(s-\theta)\right)\right. \\
& \left.-g_{j}\left(\psi_{j}(s-\theta)\right)\right] d \theta \\
& \times \int_{0}^{\infty} k_{i l}(\theta) g_{l}\left(\phi_{l}(s-\theta)\right) d \theta \\
& +\sum_{j=1}^{n} \sum_{l=1}^{n} b_{i j l}(s) \int_{0}^{\infty} k_{i j}(\theta) g_{j}\left(\psi_{j}(s-\theta)\right) d \theta \\
& \times \int_{0}^{\infty} k_{i l}(\theta) \\
& \times\left[g_{l}\left(\phi_{l}(s-\theta)\right)\right. \\
& \left.\left.\left.-g_{l}\left(\psi_{l}(s-\theta)\right)\right] d \theta\right] d s \mid\right\} \\
& \leq \sup _{t \in \mathbb{R}} \max _{1 \leq i \leq n}\left\{\int_{-\infty}^{t} e^{-\int_{s}^{t} c_{i}(u) d u}\right. \\
& +\sum_{j=1}^{n} \alpha_{i j}(t) \\
& \times \int_{0}^{\infty} \beta_{i j}(\theta) \\
& \times\left[h_{j}\left(\phi_{j}^{\prime}(t-\theta)\right)-h_{j}\left(\psi_{j}^{\prime}(t-\theta)\right)\right] d \theta \\
& +\sum_{j=1}^{n} \sum_{l=1}^{n} b_{i j l}(t) \\
& \times \int_{0}^{\infty} k_{i j}(\theta)\left[g_{j}\left(\phi_{j}(t-\theta)\right)\right. \\
& \left.-g_{j}\left(\psi_{j}(t-\theta)\right)\right] d \theta \\
& \times \int_{0}^{\infty} k_{i l}(\theta) g_{l}\left(\phi_{l}(t-\theta)\right) d \theta \\
& +\sum_{j=1}^{n} \sum_{l=1}^{n} b_{i j l}(t) \\
& \times\left[\sum_{j=1}^{n} \overline{a_{i j}} d_{i j}^{M} \epsilon_{j}\|\phi-\psi\|_{\infty}\right. \\
& \times \int_{0}^{\infty} k_{i j}(\theta) g_{j}\left(\psi_{j}(t-\theta)\right) d \theta \\
& \times \int_{0}^{\infty} k_{i l}(\theta)\left[g_{l}\left(\phi_{l}(t-\theta)\right)\right. \\
& +\sum_{j=1}^{n} \overline{\alpha_{i j}} \beta_{i j}^{M} \vartheta_{j}\left\|(\phi-\psi)^{\prime}\right\|_{\infty} \\
& \left.-g_{l}\left(\psi_{l}(t-\theta)\right)\right] d \theta \\
& \left.\left.+2 \sum_{j=1}^{n} \sum_{l=1}^{n} \overline{b_{i j l}} G_{l} \varepsilon_{j} k_{i j}^{M} k_{i l}^{M}\|\phi-\psi\|_{\infty}\right] d s\right\} \\
& -\int_{-\infty}^{t} c_{i}(t) e^{-\int_{s}^{t} c_{i}(u) d u} \\
& \times\left[\sum_{j=1}^{n} a_{i j}(s)\right. \\
& \leq \sup _{t \in \mathbb{R}} \max _{1 \leq i \leq n}\left\{\int_{-\infty}^{t} e^{-c_{i}^{m}(t-s)}\right. \\
& \times \sum_{j=1}^{n}\left[\overline{a_{i j}} d_{i j}^{M} \epsilon_{j}+\bar{\alpha}_{i j} \beta_{i j}^{M} \vartheta_{j}\right. \\
& \times \int_{0}^{\infty} d_{i j}(\theta)\left[f_{j}\left(\phi_{j}(s-\theta)\right)\right. \\
& \left.-f_{j}\left(\psi_{j}(s-\theta)\right)\right] d \theta \\
& \left.\left.+2 \sum_{l=1}^{n} \overline{b_{i j l}} G_{l} \varepsilon_{j} k_{i j}^{M} k_{i l}^{M}\right] d s\|\phi-\psi\|_{\infty}^{1}\right\} \\
& +\sum_{j=1}^{n} \alpha_{i j}(s)
\end{aligned}
$$




$$
\begin{aligned}
& \times \int_{0}^{\infty} \beta_{i j}(\theta) \\
& \times\left[h_{j}\left(\phi_{j}^{\prime}(s-\theta)\right)\right. \\
& \left.-h_{j}\left(\psi_{j}^{\prime}(s-\theta)\right)\right] d \theta \\
& +\sum_{j=1}^{n} \sum_{l=1}^{n} b_{i j l}(s) \\
& \times \int_{0}^{\infty} k_{i j}(\theta) \\
& \times\left[g_{j}\left(\phi_{j}(s-\theta)\right)\right. \\
& \left.-g_{j}\left(\psi_{j}(s-\theta)\right)\right] d \theta \\
& \times \int_{0}^{\infty} k_{i l}(\theta) g_{l}\left(\phi_{l}(s-\theta)\right) d \theta \\
& +\sum_{j=1}^{n} \sum_{l=1}^{n} b_{i j l}(s) \\
& \times \int_{0}^{\infty} k_{i j}(\theta) g_{j}\left(\psi_{j}(s-\theta)\right) d \theta \\
& \times \int_{0}^{\infty} k_{i l}(\theta) \\
& \times\left[g_{l}\left(\phi_{l}(s-\theta)\right)-g_{l}\right. \\
& \left.\left.\left.\times\left(\psi_{l}(s-\theta)\right)\right] d \theta\right] d s \mid\right\} \\
& \leq \max _{1 \leq i \leq n}\left\{\left(1+\frac{c_{i}^{M}}{c_{i}^{m}}\right)\right. \\
& \times\left[\sum _ { j = 1 } ^ { n } \left(\overline{a_{i j}} d_{i j}^{M} \epsilon_{j}+\overline{\alpha_{i j}} \beta_{i j}^{M} \vartheta_{j}\right.\right. \\
& \left.\left.\left.+2 \sum_{l=1}^{n} \overline{b_{i j l}} G_{l} \varepsilon_{j} k_{i j}^{M} k_{i l}^{M}\right)\right]\right\}\|\phi-\psi\|_{\infty}^{1} \\
& \leq \xi\|\phi-\psi\|_{\infty}^{1} .
\end{aligned}
$$

Thus,

$$
\|T \phi-T \psi\|_{\infty}^{1} \leq \xi\|\phi-\psi\|_{\infty}^{1} .
$$

Notice that $\xi<1$; it means that the mapping $T$ is a contraction mapping. By Banach fixed point theorem, there exists a unique fixed point $z \in E$ such that $T z=z$, which implies that system (1) has a unique weighted pseudo-almost periodic solution. This completes the proof.

Since $\left(A P^{1}\left(\mathbb{R}, \mathbb{R}^{n}\right),\|\cdot\|_{\infty}^{1}\right)$ and $\left(P A P^{1}\left(\mathbb{R}, \mathbb{R}^{n}\right),\|\cdot\|_{\infty}^{1}\right)$ are Banach spaces, we can get the following corollary.
Corollary 13. If $\left(\mathrm{H}_{1}\right)-\left(\mathrm{H}_{3}\right)$ and $\left(\mathrm{H}_{5}\right)$ hold, furthermore, assume that $I_{i}(t)(i=1,2, \ldots, n)$ are almost periodic functions; then there exists a unique continuously differentiable almost periodic solution of system (1) in the region

$$
E=\left\{\phi \mid \phi \in A P^{1}\left(\mathbb{R}, \mathbb{R}^{n}\right),\left\|\phi-\phi_{0}\right\|_{\infty}^{1} \leq \frac{A \xi+B}{1-\xi}\right\},
$$

where $A P^{1}\left(\mathbb{R}, \mathbb{R}^{n}\right)=\left\{f=\left(f_{1}(t), f_{2}(t), \ldots, f_{n}(t)\right)^{T}\right.$ । $\left.f_{i}(t), f_{i}^{\prime}(t) \in A P(\mathbb{R}, \mathbb{R}), i=1,2, \ldots, n\right\}$.

Corollary 14. If $\left(\mathrm{H}_{1}\right)-\left(\mathrm{H}_{3}\right)$ and $\left(\mathrm{H}_{5}\right)$ hold, furthermore, assume that $I_{i}(t)(i=1,2, \ldots, n)$ are pseudo-almost periodic functions; then there exists a unique continuously differentiable pseudo-almost periodic solution of system (1) in the region

$$
E=\left\{\phi \mid \phi \in P A P^{1}\left(\mathbb{R}, \mathbb{R}^{n}\right),\left\|\phi-\phi_{0}\right\|_{\infty}^{1} \leq \frac{A \xi+B}{1-\xi}\right\},
$$

where $P A P^{1}\left(\mathbb{R}, \mathbb{R}^{n}\right)=\left\{f=\left(f_{1}(t), f_{2}(t), \ldots, f_{n}(t)\right)^{T}\right.$ $\left.f_{i}(t), f_{i}^{\prime}(t) \in \operatorname{PAP}(\mathbb{R}, \mathbb{R}), i=1,2, \ldots, n\right\}$.

\section{Global Exponential Stability of Weighted Pseudo-Almost Periodic Solution}

Definition 15. Fix $\mu \in \mathbb{U}_{\infty}^{\text {Inv }}$. The weighted pseudo-almost periodic solution

$$
x^{*}(t)=\left(x_{1}^{*}(t), x_{2}^{*}(t), \ldots, x_{n}^{*}(t)\right)^{T}
$$

of system (1) with initial value $\phi^{*}(t)=$ $\left(\phi_{1}^{*}(t), \phi_{2}^{*}(t), \ldots, \phi_{n}^{*}(t)\right)^{T}$ is said to be globally exponentially stable. If there exist constants $\lambda>0$ and $M \geq 1$ such that for every solution $x(t)=\left(x_{1}(t), x_{2}(t), \ldots, x_{n}(t)\right)^{T}$ of system (1) with any initial value $\phi(t)=\left(\phi_{1}(t), \phi_{2}(t), \ldots, \phi_{n}(t)\right)^{T}$ satisfies

$$
\begin{array}{r}
\left\|x(t)-x^{*}(t)\right\|_{1} \\
=\max \left\{\left\|x(t)-x^{*}(t)\right\|,\left\|x^{\prime}(t)-x^{*^{\prime}}(t)\right\|\right\} \leq M\|\psi\|_{\infty}^{1} e^{-\lambda t}, \\
\forall t>0,
\end{array}
$$

where

$$
\begin{array}{r}
\|\psi\|_{\infty}^{1}=\max \left\{\sup _{t \in(-\infty, 0]} \max _{1 \leq i \leq n}\left|\phi_{i}(t)-\phi_{i}^{*}(t)\right|,\right. \\
\left.\sup _{t \in(-\infty, 0]} \max _{1 \leq i \leq n}\left|\phi_{i}^{\prime}(t)-\left(\phi_{i}^{*}\right)^{\prime}(t)\right|\right\} .
\end{array}
$$

Theorem 16. Fix $\mu \in \mathbb{U}_{\infty}^{I n v}$. If conditions $\left(H_{1}\right)-\left(H_{5}\right)$ hold, then system (1) has a unique continuously differentiable weighted 
pseudo-almost periodic solution $z(t)$ which is globally exponentially stable.

Proof. It follows from Theorem 12 that system (1) has a unique weighted pseudo-almost periodic solution

$$
x^{*}(t)=\left(x_{1}^{*}(t), x_{2}^{*}(t), \ldots, x_{n}^{*}(t)\right)^{T} \in E
$$

with initial value $\phi^{*}(t)=\left(\phi_{1}^{*}(t), \phi_{2}^{*}(t), \ldots, \phi_{n}^{*}(t)\right)^{T}$. Let $x(t)=$ $\left(x_{1}(t), x_{2}(t), \ldots, x_{n}(t)\right)^{T}$ be an arbitrary solution of system (1) with initial value $\phi(t)=\left(\phi_{1}(t), \phi_{2}(t), \ldots, \phi_{n}(t)\right)^{T}$. Let $y_{i}(t)=$ $x_{i}(t)-x_{i}^{*}(t), \psi_{i}(t)=\phi(t)-\phi_{i}^{*}(t), i=1,2, \ldots, n$; then

$$
\begin{aligned}
& y_{i}^{\prime}(s)+c_{i}(s) y_{i}(s) \\
& =\sum_{j=1}^{n} a_{i j}(s) \\
& \quad \times \int_{0}^{\infty} d_{i j}(\theta)\left[f_{j}\left(y_{j}(s-\theta)+x_{j}^{*}(s-\theta)\right)\right. \\
& \left.\left.+\sum_{j=1}^{n} \alpha_{i j}(s) \quad-x_{j}^{*}(s-\theta)\right)\right] d \theta \\
& \times \int_{0}^{\infty} \beta_{i j}(\theta)\left[h_{j}\left(y_{j}^{\prime}(s-\theta)+\left(x_{j}^{*}\right)^{\prime}(s-\theta)\right)\right. \\
& \left.-h_{j}\left(\left(x_{j}^{*}\right)^{\prime}(s-\theta)\right)\right] d \theta
\end{aligned}
$$$$
+\sum_{j=1}^{n} \sum_{l=1}^{n} b_{i j l}(s)
$$

$$
\begin{aligned}
& \times\left[\left(\int_{0}^{\infty} k_{i j}(\theta)\right.\right. \\
& \times\left[g_{j}\left(y_{j}(s-\theta)+x_{j}^{*}(s-\theta)\right)\right. \\
& \left.\quad-g_{j}\left(x_{j}^{*}(s-\theta)\right)\right] d \theta \\
& \left.\times \int_{0}^{\infty} k_{i l}(\theta) g_{l}\left(y_{l}(s-\theta)+x_{l}^{*}(s-\theta)\right) d \theta\right) \\
& +\left(\int_{0}^{\infty} k_{i j}(\theta) g_{j}\left(x_{j}^{*}(s-\theta)\right) d \theta\right. \\
& \quad \times \int_{0}^{\infty} k_{i l}(\theta) \\
& \quad \times\left[g_{l}\left(y_{l}(s-\theta)+x_{l}^{*}(s-\theta)\right)\right. \\
& \left.\left.\left.\quad-g_{l}\left(x_{l}^{*}(s-\theta)\right)\right] d \theta\right)\right]
\end{aligned}
$$

where $i=1,2, \ldots, n$. Let $H_{i}$ and $H_{i}^{*}$ be defined by

$$
\begin{aligned}
& H_{i}(\eta) \\
& =c_{i}^{m}-\eta \\
& -\sum_{j=1}^{n}\left[\overline{a_{i j}} \epsilon_{j} \int_{0}^{\infty}\left|d_{i j}(\theta)\right| e^{\eta \theta} d \theta+\overline{\alpha_{i j}} \vartheta_{j} \int_{0}^{\infty}\left|\beta_{i j}(\theta)\right| e^{\eta \theta} d \theta\right. \\
& \left.+2 \varepsilon_{j} \sum_{l=1}^{n} \overline{b_{i j l}} G_{l} k_{i l}^{M} \int_{0}^{\infty}\left|k_{i j}(\theta)\right| e^{\eta \theta} d \theta\right], \\
& =c_{i}^{m}-\eta-\left(c_{i}^{M}+c_{i}^{m}\right) \\
& \times \sum_{j=1}^{n}\left[\overline{a_{i j}} \epsilon_{j} \int_{0}^{\infty}\left|d_{i j}(\theta)\right| e^{\eta \theta} d \theta+\overline{\alpha_{i j}} \vartheta_{j} \int_{0}^{\infty}\left|\beta_{i j}(\theta)\right| e^{\eta \theta} d \theta\right. \\
& \left.+2 \varepsilon_{j} \sum_{l=1}^{n} \overline{b_{i j l}} G_{l} k_{i l}^{M} \int_{0}^{\infty}\left|k_{i j}(\theta)\right| e^{\eta \theta} d \theta\right],
\end{aligned}
$$

where $i=1,2, \ldots, n, \eta \in[0, \infty)$. By $\left(\mathrm{H}_{4}\right)$, we obtain that $H_{i}(0)$

$$
\begin{gathered}
=c_{i}^{m} \\
-\sum_{j=1}^{n}\left[\int _ { 0 } ^ { \infty } \left(\overline{a_{i j}} \epsilon_{j}\left|d_{i j}(\theta)\right|+\overline{\alpha_{i j}} \vartheta_{j}\left|\beta_{i j}(\theta)\right|\right.\right. \\
\left.\left.+2 \varepsilon_{j} \sum_{l=1}^{n} \overline{b_{i j l}} G_{l} k_{i l}^{M}\left|k_{i j}(\theta)\right|\right) d \theta\right] \\
\geq c_{i}^{m}-\sum_{j=1}^{n}\left[\overline{a_{i j}} d_{i j}^{M} \epsilon_{j}+\overline{\alpha_{i j}} \beta_{i j}^{M} \vartheta_{j}+2 \varepsilon_{j} \sum_{l=1}^{n} \overline{b_{i j l}} G_{l} k_{i l}^{M} k_{i j}^{M}\right]>0, \\
i=1,2, \ldots, n,
\end{gathered}
$$

$H_{i}^{*}(0)$

$$
\begin{aligned}
& =c_{i}^{m}-\left(c_{i}^{M}+c_{i}^{m}\right) \\
& \times \sum_{j=1}^{n}\left[\overline{a_{i j}} \epsilon_{j} \int_{0}^{\infty}\left|d_{i j}(\theta)\right| d \theta+\overline{\alpha_{i j}} \vartheta_{j} \int_{0}^{\infty}\left|\beta_{i j}(\theta)\right| d \theta\right. \\
& \left.\quad+2 \varepsilon_{j} \sum_{l=1}^{n} \overline{b_{i j l}} G_{l} k_{i l}^{M} \int_{0}^{\infty}\left|k_{i j}(\theta)\right| d \theta\right] \\
& \geq c_{i}^{m}-\left(c_{i}^{M}+c_{i}^{m}\right) \\
& \quad \times \sum_{j=1}^{n}\left[\overline{a_{i j}} d_{i j}^{M} \epsilon_{j}+\overline{\alpha_{i j}} \beta_{i j}^{M} \vartheta_{j}+2 \varepsilon_{j} \sum_{l=1}^{n} \overline{b_{i j l}} G_{l} k_{i l}^{M} k_{i j}^{M}\right]>0, \\
& \quad i=1,2, \ldots, n .
\end{aligned}
$$


Since $H_{i}, H_{i}^{*}$ are continuous on $[0, \infty)$ and $H_{i}(\eta), H_{i}^{*}(\eta) \rightarrow$ $-\infty$ as $\eta \rightarrow \infty$, there exist $\eta_{i}^{*}, \gamma_{i}^{*}>0$ such that $H_{i}\left(\eta_{i}^{*}\right)=H_{i}^{*}\left(\gamma_{i}^{*}\right)=0$, and $H_{i}\left(\eta_{i}\right)>0$ for $\eta_{i} \epsilon$ $\left(0, \eta_{i}^{*}\right), H_{i}^{*}\left(\gamma_{i}\right)>0$ for $\gamma_{i} \in\left(0, \gamma_{i}^{*}\right)$. By choosing $\gamma=$ $\min \left\{\eta_{1}^{*}, \eta_{2}^{*}, \ldots, \eta_{n}^{*}, \gamma_{1}^{*}, \gamma_{2}^{*}, \ldots, \gamma_{n}^{*}\right\}$, we have

$$
H_{i}(\gamma) \geq 0, \quad H_{i}^{*}(\gamma) \geq 0, \quad i=1,2, \ldots, n .
$$

So, we can choose a positive constant $0<\lambda<\min \left\{\gamma, \lambda_{0}\right\}$ such that $H_{i}(\lambda)>0$ and $H_{i}^{*}(\lambda)>0$, which implies that

$$
\begin{aligned}
& \frac{1}{c_{i}^{m}-\lambda} \sum_{j=1}^{n}\left[\int _ { 0 } ^ { \infty } \left(\overline{a_{i j}} \epsilon_{j}\left|d_{i j}(\theta)\right|+\overline{\alpha_{i j}} \vartheta_{j}\left|\beta_{i j}(\theta)\right|\right.\right. \\
& \left.\left.\quad+2 \varepsilon_{j} \sum_{l=1}^{n} \overline{b_{i j l}} G_{l} k_{i l}^{M}\left|k_{i j}(\theta)\right|\right) e^{\lambda \theta} d \theta\right]<1, \\
& \left(1+\frac{c_{i}^{m}}{c_{i}^{m}-\lambda}\right) \\
& \times \sum_{j=1}^{n}\left[\int _ { 0 } ^ { \infty } \left(\overline{a_{i j}} \epsilon_{j}\left|d_{i j}(\theta)\right|+\overline{\alpha_{i j}} \vartheta_{j}\left|\beta_{i j}(\theta)\right|\right.\right. \\
& \left.\left.+2 \varepsilon_{j} \sum_{l=1}^{n} \overline{b_{i j l}} G_{l} k_{i l}^{M}\left|k_{i j}(\theta)\right|\right) e^{\lambda \theta} d \theta\right]<1,
\end{aligned}
$$

where $i=1,2, \ldots, n$.

Multiplying both sides of (44) by $e^{\int_{0}^{s} c_{i}(u) d u}$ and integrating it over $[0, t]$, we have

$$
\begin{aligned}
& y_{i}(t) \\
& =y_{i}(0) e^{-\int_{0}^{t} c_{i}(u) d u} \\
& +\int_{0}^{t} e^{-\int_{s}^{t} c_{i}(u) d u} \\
& \times\left[\sum_{j=1}^{n} a_{i j}(s)\right. \\
& \times \int_{0}^{\infty} d_{i j}(\theta)\left[f_{j}\left(y_{j}(s-\theta)+x_{j}^{*}(s-\theta)\right)\right. \\
& +\sum_{j=1}^{n} \alpha_{i j}(s) \\
& \times \int_{0}^{\infty} \beta_{i j}(\theta)\left[f_{j}^{*}\left(y_{j}^{\prime}(s-\theta)\right)\right] d \theta \\
& +\sum_{j=1}^{n} \sum_{l=1}^{n} b_{i j l}(s)
\end{aligned}
$$

$$
\begin{aligned}
\times\left(\int_{0}^{\infty} k_{i j}(\theta)\right. \\
\times\left[g_{j}\left(y_{j}(s-\theta)+x_{j}^{*}(s-\theta)\right)\right. \\
\left.\quad-g_{j}\left(x_{j}^{*}(s-\theta)\right)\right] d \theta \\
\times \int_{0}^{\infty} k_{i l}(\theta) \\
\left.\quad \times g_{l}\left(y_{l}(s-\theta)+x_{l}^{*}(s-\theta)\right) d \theta\right)
\end{aligned}
$$$$
+\sum_{j=1}^{n} \sum_{l=1}^{n} b_{i j l}(s)
$$$$
\times\left(\int_{0}^{\infty} k_{i j}(\theta) g_{j}\left(x_{j}^{*}(s-\theta)\right) d \theta\right.
$$$$
\times \int_{0}^{\infty} k_{i l}(\theta)
$$$$
\times\left[g_{j}\left(y_{j}(s-\theta)+x_{j}^{*}(s-\theta)\right)\right.
$$$$
\left.\left.\left.-g_{j}\left(x_{j}^{*}(s-\theta)\right)\right] d \theta\right)\right] d s .
$$

Let

$M$

$$
\begin{aligned}
&=\max _{1 \leq i \leq n}\left\{c _ { i } ^ { m } \left(\sum _ { j = 1 } ^ { n } \left[\int _ { 0 } ^ { \infty } \left(\overline{a_{i j}} \epsilon_{j}\left|d_{i j}(\theta)\right|+\overline{\alpha_{i j}} \vartheta_{j}\left|\beta_{i j}(\theta)\right|\right.\right.\right.\right. \\
&+2 \varepsilon_{j} \sum_{l=1}^{n} \overline{b_{i j l}} G_{l} k_{i l}^{M} \\
&\left.\left.\left.\left.\times\left|k_{i j}(\theta)\right|\right) d \theta\right]\right)^{-1}\right\} .
\end{aligned}
$$

By $\left(\mathrm{H}_{4}\right)$, we have $M>1$. Thus,

$$
\begin{aligned}
& \frac{1}{M}-\frac{1}{c_{i}^{m}-\lambda} \\
& \times\left[\sum _ { j = 1 } ^ { n } \int _ { 0 } ^ { \infty } \left(\bar{a}_{i j} \epsilon_{j}\left|d_{i j}(\theta)\right|+\overline{\alpha_{i j}} \vartheta_{j}\left|\beta_{i j}(\theta)\right|\right.\right. \\
& \left.\left.+2 \varepsilon_{j} \sum_{l=1}^{n} \overline{b_{i j l}} G_{l} k_{i l}^{M}\left|k_{i j}(\theta)\right|\right) e^{\lambda \theta} d \theta\right] \leq 0, \\
& \|y(t)\|_{1}=\|\psi(t)\|_{1} \leq\|\psi\|_{\infty}^{1} \leq M\|\psi\|_{\infty}^{1} e^{-\lambda t},
\end{aligned}
$$$$
\forall t \in(-\infty, 0] \text {, }
$$ 
12

Abstract and Applied Analysis

where $\lambda>0$ as in (48). We claim that

$$
\|y(t)\|_{1} \leq M\|\psi\|_{\infty}^{1} e^{-\lambda t}, \quad t>0
$$

To prove (53), we first show that, for any $p>1$, the following inequality holds:

$$
\|y(t)\|_{1} \leq p M\|\psi\|_{\infty}^{1} e^{-\lambda t}, \quad t>0
$$

If (54) is not true, then there must be some $t_{1}>0$ and some $i, k \in\{1,2, \ldots, n\}$ such that

$$
\begin{gathered}
\left\|y\left(t_{1}\right)\right\|_{1}=\max \left\{\left\|y\left(t_{1}\right)\right\|,\left\|y^{\prime}\left(t_{1}\right)\right\|\right\} \\
=\max \left\{\left|y_{i}\left(t_{1}\right)\right|,\left|y_{\iota}^{\prime}\left(t_{1}\right)\right|\right\}=p M\|\psi\|_{\infty}^{1} e^{-\lambda t_{1}}, \\
\|y(t)\|_{1} \leq p M\|\psi\|_{\infty}^{1} e^{-\lambda t}, \quad t \in\left(-\infty, t_{1}\right] .
\end{gathered}
$$

By (48)-(51), (56), and $\left(\mathrm{H}_{2}\right)$, we get

$$
\begin{aligned}
& \left|y_{i}\left(t_{1}\right)\right| \\
& \leq e^{-\int_{0}^{t_{1}} c_{i}(u) d u}\|\psi\|_{\infty}^{1} \\
& +\int_{0}^{t_{1}} e^{-\int_{s}^{t_{1}} c_{i}(u) d u} \\
& \times\left[\sum_{j=1}^{n} \overline{a_{i j}} \epsilon_{j} \int_{0}^{\infty}\left|d_{i j}(\theta)\right|\left\|y_{j}(s-\theta)\right\| d \theta\right. \\
& \quad+\sum_{j=1}^{n} \overline{\alpha_{i j}} \vartheta_{j} \int_{0}^{\infty}\left|\beta_{i j}(\theta)\right|\left|y_{j}^{\prime}(s-\theta)\right| d \theta \\
& \quad+2 \sum_{j=1}^{n} \sum_{l=1}^{n} \overline{b_{i j l}} G_{l} k_{i l}^{M} \varepsilon_{j} \\
& \left.+\int_{0}^{-c_{i}^{m} t_{1}}\|\psi\|_{\infty}^{1} e^{-\int_{s}^{t_{1}} c_{i}(u) d u} \times \int_{0}^{\infty}\left|k_{i j}(\theta)\right|\left|y_{j}(s-\theta)\right| d \theta\right] d s
\end{aligned}
$$

$$
\begin{aligned}
& \times\left[\sum_{j=1}^{n} \overline{a_{i j}} \epsilon_{j} p M\|\psi\|_{\infty}^{1} e^{-\lambda s} \int_{0}^{\infty}\left|d_{i j}(\theta)\right| e^{\lambda \theta} d \theta\right. \\
& +\sum_{j=1}^{n} \overline{\alpha_{i j}} \vartheta_{j} p M\|\psi\|_{\infty}^{1} e^{-\lambda s} \int_{0}^{\infty}\left|\beta_{i j}(\theta)\right| e^{\lambda \theta} d \theta \\
& +2 \sum_{j=1}^{n} \sum_{l=1}^{n} \overline{b_{i j l}} G_{l} \varepsilon_{j} k_{i l}^{M} p M\|\psi\|_{\infty}^{1} e^{-\lambda s} \\
& \left.\times \int_{0}^{\infty}\left|k_{i j}(\theta)\right| e^{\lambda \theta} d \theta\right] d s \\
& \leq e^{-c_{i}^{m} t_{1}}\|\psi\|_{\infty}^{1}+p M\|\psi\|_{\infty}^{1} \\
& \times\left[\sum_{j=1}^{n} \int_{0}^{\infty}\left(\overline{a_{i j}} \epsilon_{j}\left|d_{i j}(\theta)\right|+\overline{\alpha_{i j}} \vartheta_{j}\left|\beta_{i j}(\theta)\right|\right) e^{\lambda \theta} d \theta\right. \\
& \left.+2 \sum_{j=1}^{n} \sum_{l=1}^{n} \overline{b_{i j l}} G_{l} \varepsilon_{j} k_{i l}^{M} \int_{0}^{\infty}\left|k_{i j}(\theta)\right| e^{\lambda \theta} d \theta\right] \\
& \times \int_{0}^{t_{1}} e^{-c_{i}^{m}\left(t_{1}-s\right)-\lambda s} d s \\
& =e^{-c_{i}^{m} t_{1}}\|\psi\|_{\infty}^{1}+p M\|\psi\|_{\mathbb{X}} \\
& \times \sum_{j=1}^{n}\left[\int_{0}^{\infty}\left(\overline{a_{i j}} \epsilon_{j}\left|d_{i j}(\theta)\right|+\overline{\alpha_{i j}} \vartheta_{j}\left|\beta_{i j}(\theta)\right|\right) e^{\lambda \theta} d \theta\right. \\
& \left.+2 \varepsilon_{j} \sum_{l=1}^{n} \overline{b_{i j l}} G_{l} k_{i l}^{M} \int_{0}^{\infty}\left|k_{i j}(\theta)\right| e^{\lambda \theta} d \theta\right] \\
& \times \frac{e^{-\lambda t_{1}}}{c_{i}^{m}-\lambda}\left(1-e^{\left(\lambda-c_{i}^{m}\right) t_{1}}\right) \\
& =p M\|\psi\|_{\infty}^{1} \\
& \times e^{-\lambda t_{1}}\left\{\frac{1}{p M} e^{\left(\lambda-c_{i}^{m}\right) t_{1}}+\frac{1}{c_{i}^{m}-\lambda}\left(1-e^{\left(\lambda-c_{i}^{m}\right) t_{1}}\right)\right. \\
& \times\left[\sum _ { j = 1 } ^ { n } \int _ { 0 } ^ { \infty } \left(\overline{a_{i j}} \epsilon_{j}\left|d_{i j}(\theta)\right|+\overline{\alpha_{i j}} \vartheta_{j}\left|\beta_{i j}(\theta)\right|\right.\right. \\
& \left.+2 \varepsilon_{j} \sum_{l=1}^{n} \overline{b_{i j l}} G_{l} k_{i l}^{M}\left|k_{i j}(\theta)\right|\right) \\
& \left.\left.\times e^{\lambda \theta} d \theta\right]\right\} \\
& <p M\|\psi\|_{\infty}^{1} \\
& \times e^{-\lambda t_{1}}\left\{\frac{1}{M} e^{\left(\lambda-c_{i}^{m}\right) t_{1}}+\frac{1}{c_{i}^{m}-\lambda}\left(1-e^{\left(\lambda-c_{i}^{m}\right) t_{1}}\right)\right.
\end{aligned}
$$




$$
\begin{aligned}
& \times\left[\sum _ { j = 1 } ^ { n } \int _ { 0 } ^ { \infty } \left(\overline{a_{i j}} \epsilon_{j}\left|d_{i j}(\theta)\right|+\overline{\alpha_{i j}} \vartheta_{j}\left|\beta_{i j}(\theta)\right|\right.\right. \\
& \left.+2 \varepsilon_{j} \sum_{l=1}^{n} \overline{b_{i j l}} G_{l} k_{i l}^{M}\left|k_{i j}(\theta)\right|\right) \\
& \left.\left.\times e^{\lambda \theta} d \theta\right]\right\} \\
& =p M\|\psi\|_{\infty}^{1} \\
& \times e^{-\lambda t_{1}}\left(\frac{1}{M}-\frac{1}{c_{i}^{m}-\lambda}\right. \\
& \times \sum_{j=1}^{n}\left[\int_{0}^{\infty}\left(\overline{a_{i j}} \epsilon_{j}\left|d_{i j}(\theta)\right|+\overline{\alpha_{i j}} \vartheta_{j}\left|\beta_{i j}(\theta)\right|\right)\right. \\
& \times e^{\lambda \theta} d \theta \\
& \left.\left.+2 \varepsilon_{j} \sum_{l=1}^{n} \overline{b_{i j l}} G_{l} k_{i l}^{M} \int_{0}^{\infty}\left|k_{i j}(\theta)\right| e^{\lambda \theta} d \theta\right]\right) \\
& \times e^{\left(\lambda-c_{i}^{m}\right) t_{1}} \\
& +\frac{1}{c_{i}^{m}-\lambda} \sum_{j=1}^{n}\left[\int _ { 0 } ^ { \infty } \left(\overline{a_{i j}} \epsilon_{j}\left|d_{i j}(\theta)\right|+\overline{\alpha_{i j}} \vartheta_{j}\left|\beta_{i j}(\theta)\right|\right.\right. \\
& \left.\left.+2 \varepsilon_{j} \sum_{l=1}^{n} \overline{b_{i j l}} G_{l} k_{i l}^{M}\left|k_{i j}(\theta)\right|\right) d \theta\right] \\
& \leq p M\|\psi\|_{\infty}^{1} e^{-\lambda t_{1}} \frac{1}{c_{i}^{m}-\lambda} \\
& \times \sum_{j=1}^{n}\left[\int _ { 0 } ^ { \infty } \left(\overline{a_{i j}} \epsilon_{j}\left|d_{i j}(\theta)\right|+\overline{\alpha_{i j}} \vartheta_{j}\left|\beta_{i j}(\theta)\right|\right.\right. \\
& \left.\left.+2 \varepsilon_{j} \sum_{l=1}^{n} \overline{b_{i j l}} G_{l} k_{i l}^{M}\left|k_{i j}(\theta)\right|\right) d \theta\right] \\
& <p M\|\psi\|_{\infty}^{1} e^{-\lambda t_{1}} .
\end{aligned}
$$

Direct differentiation of (49) gives

$y_{i}^{\prime}(t)$

$$
\begin{gathered}
=-c_{i}(t) \phi_{i}(0) e^{-\int_{0}^{t} c_{i}(u) d u} \\
+\sum_{j=1}^{n} a_{i j}(t) \int_{0}^{\infty} d_{i j}(\theta)\left[f_{j}\left(y_{j}(t-\theta)+x_{j}^{*}(t-\theta)\right)\right. \\
\left.\quad-f_{j}\left(x_{j}^{*}(t-\theta)\right)\right] d \theta
\end{gathered}
$$

$$
\begin{gathered}
+\sum_{j=1}^{n} \alpha_{i j}(t) \int_{0}^{\infty} \beta_{i j}(\theta)\left[h_{j}\left(y_{j}^{\prime}(t-\theta)+\left(x_{j}^{*}\right)^{\prime}(t-\theta)\right)\right. \\
\left.-h_{j}\left(\left(x_{j}^{*}\right)^{\prime}(t-\theta)\right)\right] d \theta \\
+\sum_{j=1}^{n} \sum_{l=1}^{n} b_{i j l}(t)\left(\int_{0}^{\infty} k_{i j}(\theta)\right. \\
\times\left[g_{j}\left(y_{j}(t-\theta)+x_{j}^{*}(t-\theta)\right)\right. \\
\left.-g_{j}\left(x_{j}^{*}(t-\theta)\right)\right] d \theta \\
\times \int_{0}^{\infty} k_{i l}(\theta) g_{l} \\
\left.\times\left(y_{l}(t-\theta)+x_{l}^{*}(t-\theta)\right) d \theta\right)
\end{gathered}
$$$$
+\sum_{j=1}^{n} \sum_{l=1}^{n} b_{i j l}(t)\left(\int_{0}^{\infty} k_{i j}(\theta) g_{j}\left(x_{j}^{*}(t-\theta)\right) d \theta\right.
$$$$
\times \int_{0}^{\infty} k_{i l}(\theta)\left[g_{l}\left(y_{l}(t-\theta)+x_{l}^{*}(t-\theta)\right)\right.
$$$$
\left.\left.-g_{l}\left(x_{l}^{*}(t-\theta)\right)\right] d \theta\right)
$$$$
-\int_{0}^{t} c_{i}(t) e^{-\int_{s}^{t} c_{i}(u) d u}
$$$$
\times\left[\sum _ { j = 1 } ^ { n } a _ { i j } ( s ) \int _ { 0 } ^ { \infty } d _ { i j } ( \theta ) \left[f_{j}\left(y_{j}(s-\theta)+x_{j}^{*}(s-\theta)\right)\right.\right.
$$$$
\left.-f_{j}\left(x_{j}^{*}(s-\theta)\right)\right] d \theta
$$$$
+\sum_{j=1}^{n} \sum_{l=1}^{n} b_{i j l}(s)\left(\int_{0}^{\infty} k_{i j}(\theta)\right.
$$$$
\times\left[g_{j}\left(y_{j}(s-\theta)+x_{j}^{*}(s-\theta)\right)\right.
$$$$
\left.-g_{j}\left(x_{j}^{*}(s-\theta)\right)\right] d \theta
$$$$
\times \int_{0}^{\infty} k_{i l}(\theta) g_{l}
$$$$
\left.\times\left(y_{l}(s-\theta)+x_{l}^{*}(s-\theta)\right) d \theta\right)
$$$$
+\sum_{j=1}^{n} \sum_{l=1}^{n} b_{i j l}(s)\left(\int_{0}^{\infty} k_{i j}(\theta) g_{j}\left(x_{j}^{*}(s-\theta)\right) d \theta\right.
$$$$
\times \int_{0}^{\infty} k_{i l}(\theta)
$$$$
\times\left[g_{l}\left(y_{l}(s-\theta)+x_{l}^{*}(s-\theta)\right)\right.
$$$$
\left.\left.\left.-g_{l}\left(x_{l}^{*}(s-\theta)\right)\right] d \theta\right)\right] d s,
$$ 
where $i=1,2, \ldots, n$. Thus, we have by (48) and (58) and $\left(\mathrm{H}_{2}\right)$ $\left(\mathrm{H}_{3}\right)$ that

$$
\begin{aligned}
& \left|y_{\iota}^{\prime}\left(t_{1}\right)\right| \\
& \leq c_{l}^{M}\|\psi\|_{\infty}^{1} e^{-\int_{0}^{t_{1}} c_{l}(u) d u} \\
& +\sum_{j=1}^{n} \overline{a_{\iota j}} \int_{0}^{\infty}\left|d_{i j}(\theta)\right| \epsilon_{j}\left|y_{j}\left(t_{1}-\theta\right)\right| d \theta \\
& +\sum_{j=1}^{n} \overline{\alpha_{l j}} \int_{0}^{\infty}\left|\beta_{l j}(\theta)\right| \vartheta_{j}\left|y_{j}^{\prime}\left(t_{1}-\theta\right)\right| d \theta \\
& +2 \sum_{j=1}^{n} \sum_{l=1}^{n} \overline{b_{i j l}} G_{l} k_{l l}^{M} \int_{0}^{\infty}\left|k_{l j}(\theta)\right| \\
& \times \varepsilon_{j}\left|y_{j}\left(t_{1}-\theta\right)\right| d \theta \\
& +\int_{0}^{t_{1}} c_{l}^{M} e^{-c_{l}^{m}\left(t_{1}-s\right)} \\
& \times\left[\sum_{j=1}^{n} \overline{a_{\iota j}} p M\|\psi\|_{\infty}^{1} e^{-\lambda t_{1}}\right. \\
& \times \int_{0}^{\infty}\left|d_{i j}(\theta)\right| \epsilon_{j}\left|y_{j}(s-\theta)\right| d \theta \\
& +\sum_{j=1}^{n} \overline{\alpha_{t j}} p M\|\psi\|_{\infty}^{1} e^{-\lambda t_{1}} \\
& \times \int_{0}^{\infty}\left|\beta_{l j}(\theta)\right| \vartheta_{j}\left|y_{j}^{\prime}(s-\theta)\right| d \theta \\
& +2 \sum_{j=1}^{n} \sum_{l=1}^{n} \overline{b_{i j l}} G_{l} k_{l l}^{M} p M\|\psi\|_{\mathbb{X}} e^{-\lambda t_{1}} \\
& \left.\times \int_{0}^{\infty}\left|k_{\iota j}(\theta)\right| \varepsilon_{j}\left|y_{j}(s-\theta)\right| d \theta\right] d s \\
& =p M\|\psi\|_{\infty}^{1} e^{-\lambda t_{1}} \\
& \times\left\{\frac{c_{l}^{M}}{p M} e^{\left(\lambda-c_{t}^{m}\right) t_{1}}+\left(1+c_{l}^{M} \int_{0}^{t_{1}} e^{\left(t_{1}-s\right)\left(\lambda-c_{t}^{m}\right)} d s\right)\right. \\
& \times\left[\sum _ { j = 1 } ^ { n } \int _ { 0 } ^ { \infty } \left(\overline{a_{t j}} \epsilon_{j}\left|d_{t j}(\theta)\right|+\overline{\alpha_{t j}} \vartheta_{j}\left|\beta_{t j}(\theta)\right|\right.\right. \\
& \left.+2 \sum_{j=1}^{n} \sum_{l=1}^{n} \overline{b_{l j l}} G_{l} \varepsilon_{j} k_{l l}^{M}\left|k_{l j}(\theta)\right|\right) \\
& \left.\left.\times e^{\lambda \theta} d \theta\right]\right\} \\
& <p M\|\psi\|_{\infty}^{1} e^{-\lambda t_{1}}
\end{aligned}
$$

$$
\begin{gathered}
\times\left\{\frac{c_{l}^{M}}{M} e^{\left(\lambda-c_{l}^{m}\right) t_{1}}+\left(1+\frac{c_{l}^{M}}{c_{l}^{m}-\lambda}\left(1-e^{\left(\lambda-c_{l}^{m}\right) t_{1}}\right)\right)\right. \\
\times\left[\sum _ { j = 1 } ^ { n } \int _ { 0 } ^ { \infty } \left(\overline{a_{l j}} \epsilon_{j}\left|d_{t j}(\theta)\right|+\overline{\alpha_{l j}} \vartheta_{j}\left|\beta_{l j}(\theta)\right|\right.\right. \\
\left.+2 \sum_{j=1}^{n} \sum_{l=1}^{n} \overline{b_{i j l}} G_{l} \varepsilon_{j} k_{l l}^{M}\left|k_{l j}(\theta)\right|\right) \\
\left.\times e^{\lambda \theta} d \theta\right]
\end{gathered}
$$$$
=p M\|\psi\|_{\infty}^{1} e^{-\lambda t_{1}}
$$$$
\times\left\{\left(\frac{1}{M}-\frac{1}{c_{l}^{m}-\lambda}\right.\right.
$$$$
\times\left[\sum_{j=1}^{n} \int_{0}^{\infty}\left(\overline{a_{i j}} \epsilon_{j}\left|d_{i j}(\theta)\right|+\overline{\alpha_{i j}} \vartheta_{j}\left|\beta_{l j}(\theta)\right|\right) e^{\lambda \theta} d \theta\right.
$$$$
+2 \sum_{j=1}^{n} \sum_{l=1}^{n} \overline{b_{i j l}} G_{l} \varepsilon_{j} k_{l l}^{M}
$$$$
\left.\left.\times \int_{0}^{\infty}\left|k_{i j}(\theta)\right| e^{\lambda \theta} d \theta\right]\right)
$$$$
\times c_{l}^{M} e^{\left(\lambda-c_{l}^{m}\right) t_{1}}
$$$$
+\left(1+\frac{c_{l}^{M}}{c_{l}^{m}-\lambda}\right)
$$$$
\times\left[\sum _ { j = 1 } ^ { n } \int _ { 0 } ^ { \infty } \left(\overline{a_{l j}} \epsilon_{j}\left|d_{i j}(\theta)\right|+\overline{\alpha_{l j}} \vartheta_{j}\left|\beta_{t j}(\theta)\right|\right.\right.
$$$$
\left.+2 \sum_{j=1}^{n} \sum_{l=1}^{n} \overline{b_{i j l}} G_{l} \varepsilon_{j} k_{l l}^{M}\left|k_{t j}(\theta)\right|\right)
$$$$
\left.\left.\times e^{\lambda \theta} d \theta\right]\right\}
$$$$
<p M\|\psi\|_{\infty}^{1} e^{-\lambda t_{1}}\left(1+\frac{c_{l}^{M}}{c_{l}^{m}-\lambda}\right)
$$$$
\times\left[\sum_{j=1}^{n} \int_{0}^{\infty}\left(\overline{a_{l j}} \epsilon_{j}\left|d_{i j}(\theta)\right|+\overline{\alpha_{i j}} \vartheta_{j}\left|\beta_{i j}(\theta)\right|\right) e^{\lambda \theta} d \theta\right.
$$$$
\left.+2 \sum_{j=1}^{n} \sum_{l=1}^{n} \overline{b_{i j l}} G_{l} \varepsilon_{j} k_{l l}^{M} \int_{0}^{\infty}\left|k_{l j}(\theta)\right| d \theta\right]
$$$$
<p M\|\psi\|_{\infty}^{1} e^{-\lambda t_{1}} .
$$ 
In view of (57) and (59), we obtain

$$
\left\|y\left(t_{1}\right)\right\|_{1}<p M\|\psi\|_{\infty}^{1} e^{-\lambda t_{1}}
$$

which contradicts the equality (55), and so (54) holds. Letting $p \rightarrow 1$, (53) holds. Hence, the weighted pseudo-almost periodic solution of system (1) is globally exponentially stable.

Corollary 17. If conditions $\left(\mathrm{H}_{1}\right)-\left(\mathrm{H}_{3}\right)$ and $\left(\mathrm{H}_{5}\right)$ hold, furthermore, assume that $I_{i}(t)(i=1,2, \ldots, n)$ are almost periodic functions; then system (1) has a unique continuously differentiable almost periodic solution which is globally exponentially stable.

Corollary 18. If conditions $\left(\mathrm{H}_{1}\right)-\left(\mathrm{H}_{3}\right)$ and $\left(\mathrm{H}_{5}\right)$ hold, furthermore, assume that $I_{i}(t)(i=1,2, \ldots, n)$ are pseudo-almost periodic functions; then system (1) has a unique continuously differentiable pseudo-almost periodic solution which is globally exponentially stable.

\section{An Example}

In this section, we give one example to illustrate our result. Consider the weight $\mu(t)=e^{|t|}$ and let

$$
\begin{aligned}
& f_{1}(x)=f_{2}(x)=\frac{\sin \left(\left(1 / 2^{1 / 2}\right) x\right)}{10}, \\
& h_{1}(x)=h_{2}(x)=\frac{\sin \left(\left(1 / 2^{3 / 2}\right) x\right)}{20}, \\
& g_{1}(x)=g_{2}(x) \frac{\left|\arctan \left(\left(1 / 2^{1 / 2}\right) x\right)\right|}{20}, \\
& a_{11}(t)=1+\cos (2 \pi t), \quad a_{12}(t)=2+\cos (2 \pi t), \\
& a_{21}(t)=2+\cos (2 \pi t), \quad a_{22}(t)=3+\cos (2 \pi t), \\
& \alpha_{11}(t)=1+\sin (\pi t), \quad \alpha_{12}(t)=2+\sin ^{5}(2 \pi t), \\
& \alpha_{21}(t)=2+\cos ^{3}(2 \pi t), \quad \alpha_{22}(t)=3+\cos (\pi t), \\
& c_{1}(t)=25+5 \sin (2 \pi t), \quad c_{2}(t)=35+5 \sin (2 \pi t), \\
& I_{1}(t)=\sin (\pi t)+\sin t, \quad I_{2}(t)=\cos (\pi t)+\sin t, \\
& b_{111}(t)=b_{211}(t)=\frac{1}{4}+\frac{1}{4} \sin (\pi t), \\
& b_{112}(t)=b_{212}(t)=\frac{1}{3}+\frac{1}{3} \cos (\pi t) \text {, } \\
& b_{121}(t)=b_{221}(t)=\frac{1}{5}+\frac{1}{5} \cos (\pi t) \text {, } \\
& b_{122}(t)=b_{222}(t)=\frac{1}{6}+\frac{1}{6} \sin (\pi t), \\
& \left(d_{i j}(\theta)\right)_{2 \times 2}=\left(k_{i j}(\theta)\right)_{2 \times 2}=\left(\beta_{i j}(\theta)\right)_{2 \times 2} \\
& =\left(\begin{array}{cc}
e^{-10 \theta} & 0 \\
0 & e^{-50 \theta}
\end{array}\right) \text {. }
\end{aligned}
$$

Then system (1) has exactly one continuously differentiable weighted pseudo-almost periodic solution, which is globally exponentially stable.

Proof. By calculating,

$$
\begin{gathered}
c_{1}^{m}=20, \quad c_{1}^{M}=30, \quad c_{2}^{m}=30, \quad c_{2}^{M}=40, \\
\overline{a_{11}}=\overline{\alpha_{11}}=2, \quad \overline{a_{12}}=\overline{\alpha_{12}}=3, \quad \overline{a_{21}}=\overline{\alpha_{21}}=3, \\
\overline{a_{22}}=\overline{\alpha_{22}}=4, \quad \overline{b_{111}}=\overline{b_{211}}=\frac{1}{2}, \quad \overline{b_{112}}=\overline{b_{212}}=\frac{2}{3}, \\
\overline{b_{121}}=\overline{b_{221}}=\frac{2}{5}, \quad \overline{b_{122}}=\overline{b_{222}}=\frac{1}{3}, \quad \epsilon_{1}=\epsilon_{2}=\frac{1}{10}, \\
\vartheta_{1}=\vartheta_{2}=\frac{1}{20}, \quad \varepsilon_{1}=\varepsilon_{2}=\frac{1}{20}, \quad G_{1}=G_{2}=\frac{\pi}{40}, \\
d_{11}^{M}=k_{11}^{M}=\beta_{11}^{M}=\frac{1}{10}, \quad d_{12}^{M}=k_{12}^{M}=\beta_{12}^{M}=0, \\
d_{21}^{M}=k_{21}^{M}=\beta_{21}^{M}=0, \quad d_{22}^{M}=k_{22}^{M}=\beta_{22}^{M}=\frac{1}{50},
\end{gathered}
$$

hence we have

$$
\begin{aligned}
\xi & \max _{1 \leq i \leq 2}\left\{\max \left\{\frac{1}{c_{i}^{m}}, 1+\frac{c_{i}^{M}}{c_{i}^{m}}\right\}\right. \\
& \left.\times \sum_{j=1}^{2}\left[\overline{a_{i j}} d_{i j}^{M} \epsilon_{j}+\overline{\alpha_{i j}} \beta_{i j}^{M} \vartheta_{j}+2 \varepsilon_{j} \sum_{l=1}^{2} \overline{b_{i j l}} G_{l} k_{i j}^{M} k_{i l}^{M}\right]\right\} \\
=\max & \left\{\frac{3}{40}+\frac{\pi}{16000}, \frac{7}{100}+\frac{7 \pi}{240000}\right\}<1 .
\end{aligned}
$$

It is obvious that $\left(\mathrm{H}_{1}\right)-\left(\mathrm{H}_{5}\right)$ are satisfied. By Theorems 12 and 16, system (1) has exactly one continuously differentiable weighted pseudo-almost periodic solution, which is globally exponentially stable (see Figures 1, 2, and 3).

\section{Conclusion}

In this paper, we employ fixed point theorem and differential inequality techniques to study the existence, uniqueness, and global exponential stability of weighted pseudo-almost periodic solutions to a class of neutral type high-order Hopfield neural networks with infinitely distributed delays. Our results of this paper are new and complement previously known results, and our methods used in this paper can be used to investigate other types of neural networks such as neutral type BAM neural networks, neutral type Cohen-Grossberg neural networks, and neutral type high-order Hopfield neural networks with delays in the leakage term and so on. 


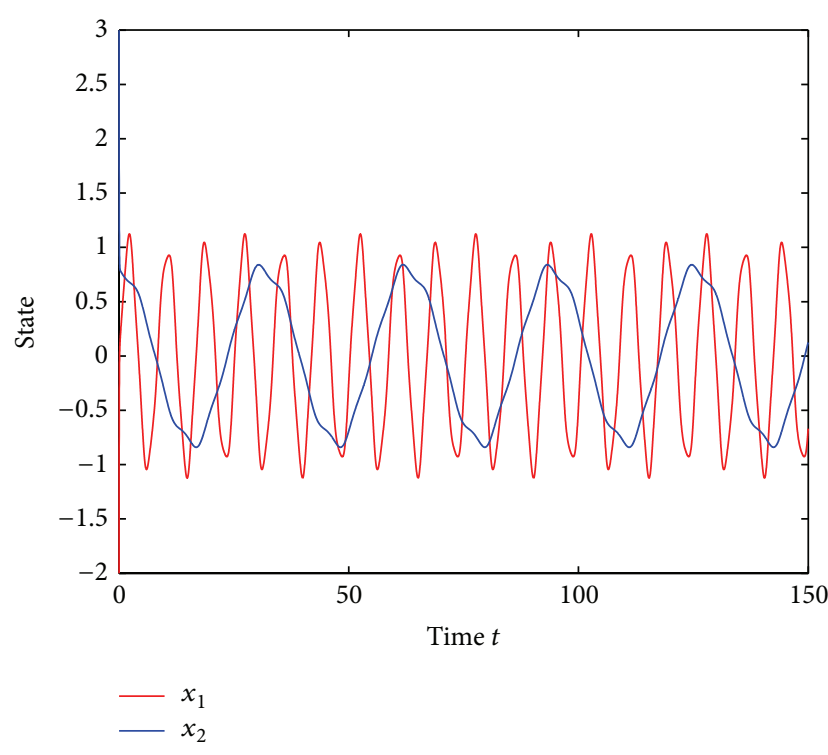

FIGURE 1: Responds of $x_{1}, x_{2}$ with time $t$.

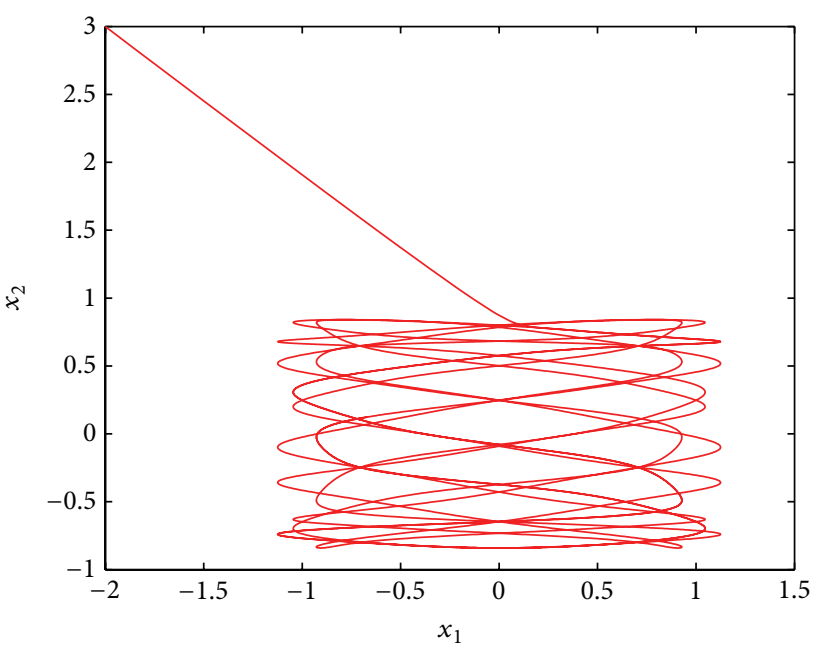

Figure 2: Responds of $x_{1}, x_{2}$.

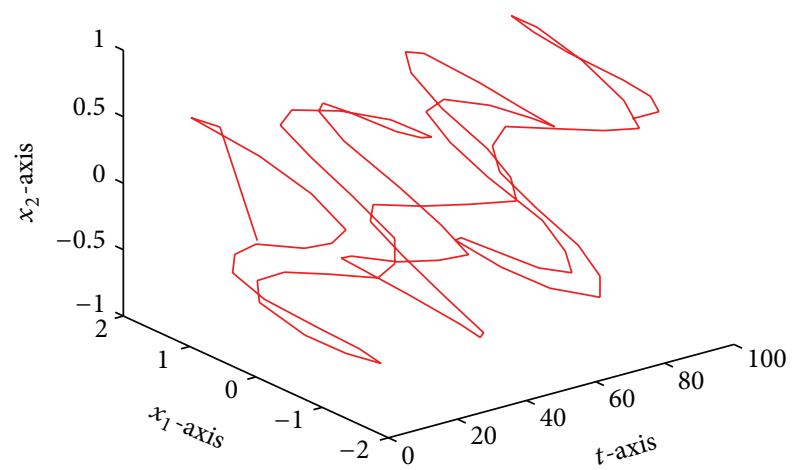

Figure 3: Responds of $x_{1}, x_{2}$ with time $t$.

\section{Conflict of Interests}

The authors declare that there is no conflict of interests regarding the publication of this paper.

\section{Acknowledgment}

This work is supported by the National Natural Sciences Foundation of China under Grant 11361072.

\section{References}

[1] Z. Wang, J. Fang, and X. Liu, "Global stability of stochastic highorder neural networks with discrete and distributed delays," Chaos, Solitons and Fractals, vol. 36, no. 2, pp. 388-396, 2008.

[2] Y. K. Li, L. Zhao, and P. Liu, "Existence and exponential stability of periodic solution of high-order hopfield neural network with delays on time scales," Discrete Dynamics in Nature and Society, vol. 2009, Article ID 573534, 18 pages, 2009.

[3] S. Mohamad, "Exponential stability in Hopfield-type neural networks with impulses," Chaos, Solitons and Fractals, vol. 32, no. 2, pp. 456-467, 2007.

[4] B. Xu, X. Liu, and X. Liao, "Global asymptotic stability of high-order Hopfield type neural networks with time delays," Computers \& Mathematics with Applications, vol. 45, no. 10-11, pp. 1729-1737, 2003.

[5] E. B. Kosmatopoulos and M. A. Christodoulou, "Structural properties of gradient recurrent high-order neural networks," IEEE Transactions on Circuits and Systems II: Analog and Digital Signal Processing, vol. 42, no. 9, pp. 592-603, 1995.

[6] F. Zhang and Y. Li, "Almost periodic solutions for higherorder Hopfield neural networks without bounded activation functions," Electronic Journal of Differential Equations, vol. 2007, no. 97, pp. 1-10, 2007.

[7] X.-Y. Lou and B.-T. Cui, "Novel global stability criteria for highorder Hopfield-type neural networks with time-varying delays," Journal of Mathematical Analysis and Applications, vol. 330, no. 1, pp. 144-158, 2007.

[8] R. Rakkiyappan, C. Pradeep, A. Vinodkumar, and F. A. Rihan, "Dynamic analysis for high-order Hopfield neural networks with leakage delay and impulsive effects," Neural Computing and Applications, vol. 22, no. 1, pp. 55-73, 2013.

[9] Q. Wang, Y. Fang, H. Li, L. Su, and B. Dai, "Anti-periodic solutions for high-order Hopfield neural networks with impulses," Neurocomputing, vol. 138, pp. 339-346, 2014.

[10] J. H. Park, C. H. Park, O. M. Kwon, and S. M. Lee, "A new stability criterion for bidirectional associative memory neural networks of neutral-type," Applied Mathematics and Computation, vol. 199, no. 2, pp. 716-722, 2008.

[11] R. Rakkiyappan and P. Balasubramaniam, "New global exponential stability results for neutral type neural networks with distributed time delays," Neurocomputing, vol. 71, no. 4-6, pp. 1039-1045, 2008.

[12] R. Rakkiyappan and P. Balasubramaniam, "LMI conditions for global asymptotic stability results for neutral-type neural networks with distributed time delays," Applied Mathematics and Computation, vol. 204, no. 1, pp. 317-324, 2008.

[13] C. Bai, "Global stability of almost periodic solutions of Hopfield neural networks with neutral time-varying delays," Applied Mathematics and Computation, vol. 203, no. 1, pp. 72-79, 2008. 
[14] B. Xiao, "Existence and uniqueness of almost periodic solutions for a class of Hopfield neural networks with neutral delays," Applied Mathematics Letters, vol. 22, no. 4, pp. 528-533, 2009.

[15] H. Xiang and J. Cao, "Almost periodic solution of CohenGrossberg neural networks with bounded and unbounded delays," Nonlinear Analysis: Real World Applications, vol. 10, no. 4, pp. 2407-2419, 2009.

[16] K. Wang and Y. Zhu, "Stability of almost periodic solution for a generalized neutral-type neural networks with delays," Neurocomputing, vol. 73, no. 16-18, pp. 3300-3307, 2010.

[17] J. Liu and G. Zong, "New delay-dependent asymptotic stability conditions concerning BAM neural networks of neutral type," Neurocomputing, vol. 72, no. 10-12, pp. 2549-2555, 2009.

[18] R. Samli and S. Arik, "New results for global stability of a class of neutral-type neural systems with time delays," Applied Mathematics and Computation, vol. 210, no. 2, pp. 564-570, 2009.

[19] R. Samidurai, S. M. Anthoni, and K. Balachandran, "Global exponential stability of neutral-type impulsive neural networks with discrete and distributed delays," Nonlinear Analysis: Hybrid Systems, vol. 4, no. 1, pp. 103-112, 2010.

[20] R. Rakkiyappan, P. Balasubramaniam, and J. Cao, "Global exponential stability results for neutral-type impulsive neural networks,' Nonlinear Analysis: Real World Applications, vol. 11, no. 1, pp. 122-130, 2010.

[21] Y. Li, L. Zhao, and X. Chen, "Existence of periodic solutions for neutral type cellular neural networks with delays," Applied Mathematical Modelling, vol. 36, no. 3, pp. 1173-1183, 2012.

[22] Y. Li and L. Yang, "Almost periodic solutions for neutral-type BAM neural networks with delays on time scales," Journal of Applied Mathematics, vol. 2013, Article ID 942309, 13 pages, 2013.

[23] Y. K. Li and Y. Q. Li, "Existence and exponential stability of almost periodic solution for neutral delay BAM neural networks with time-varying delays in leakage terms," Journal of the Franklin Institute. Engineering and Applied Mathematics, vol. 350, no. 9, pp. 2808-2825, 2013.

[24] X. Li and J. Cao, "Delay-dependent stability of neural networks of neutral type with time delay in the leakage term," Nonlinearity, vol. 23, no. 7, pp. 1709-1726, 2010.

[25] P. Balasubramaniam, G. Nagamani, and R. Rakkiyappan, "Passivity analysis for neural networks of neutral type with Markovian jumping parameters and time delay in the leakage term," Communications in Nonlinear Science and Numerical Simulation, vol. 16, no. 11, pp. 4422-4437, 2011.

[26] A. M. Fink, Almost Periodic Differential Equations, vol. 377 of Lecture Notes in Mathematics, Springer, Berlin, Germany, 1974.

[27] C. Y. He, Almost Periodic Differential Equations, Higher Education Publishing House, Beijing, China, 1992 (Chinese).

[28] T. Diagana, Almost Automorphic Type and Almost Periodic Type Functions in Abstract Spaces, Springer, Berlin, Germany, 2013.

[29] D. Ji and C. Zhang, "Translation invariance of weighted pseudo almost periodic functions and related problems," Journal of Mathematical Analysis and Applications, vol. 391, no. 2, pp. 350362, 2012. 


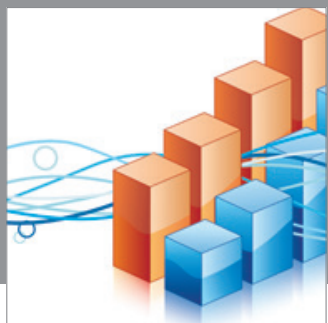

Advances in

Operations Research

mansans

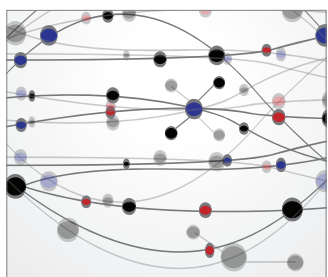

The Scientific World Journal
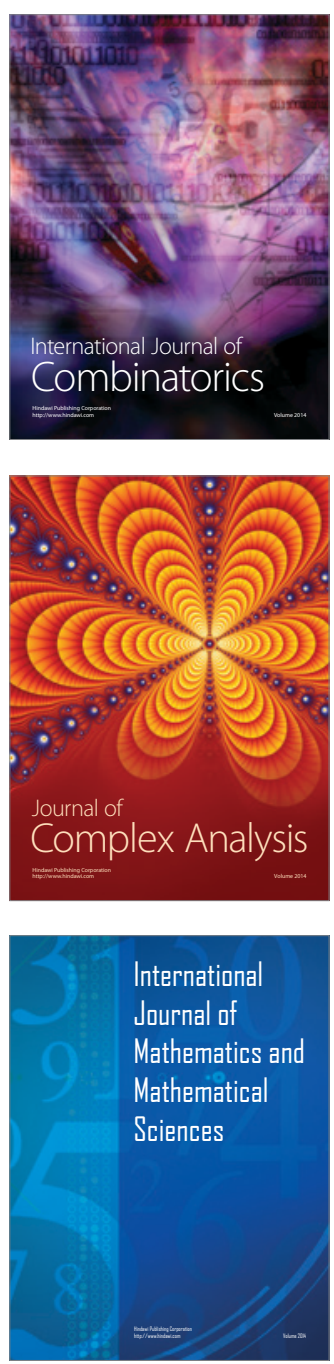
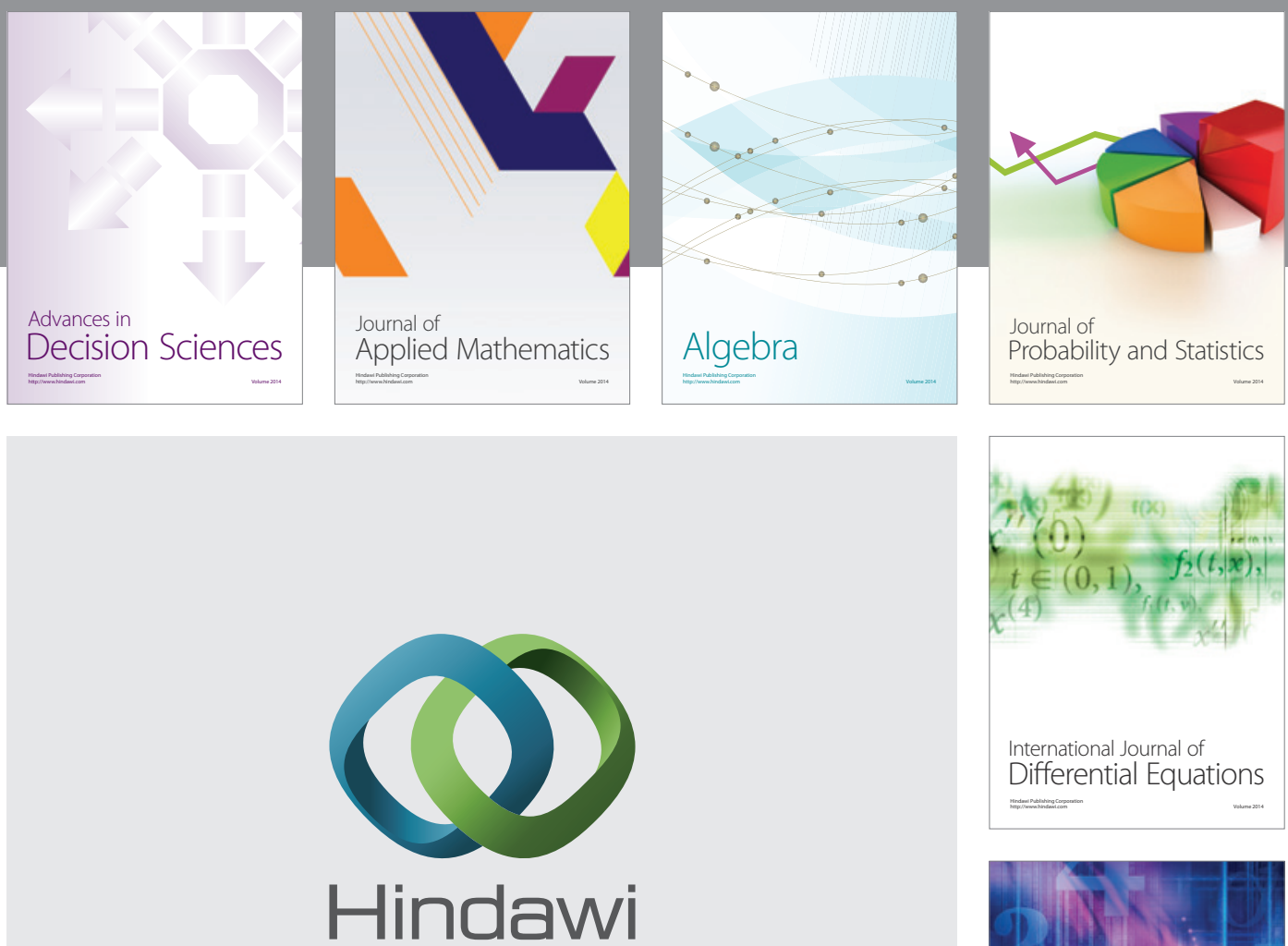

Submit your manuscripts at http://www.hindawi.com
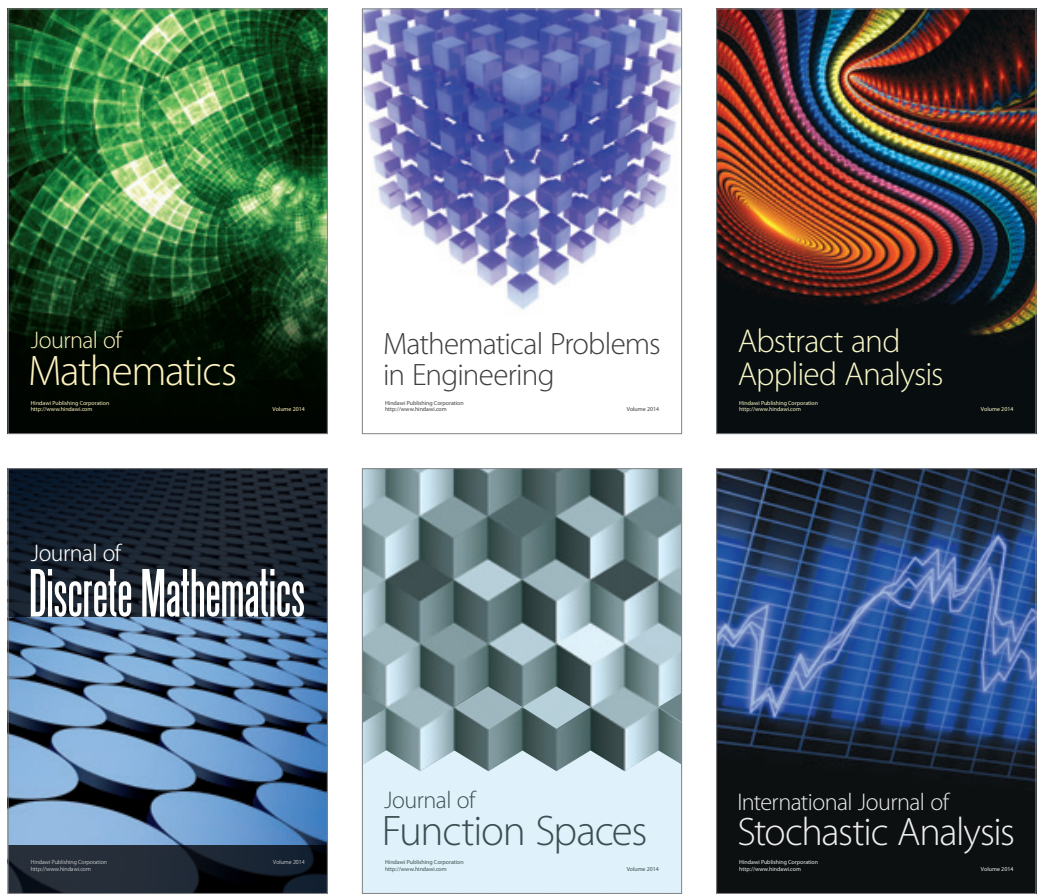

Journal of

Function Spaces

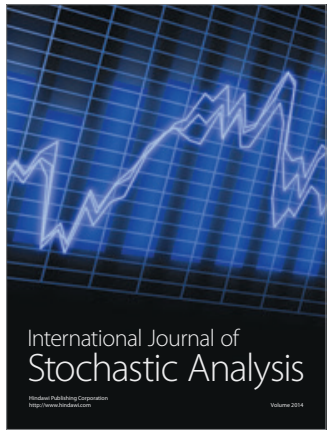

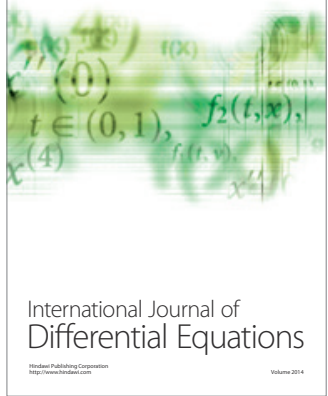
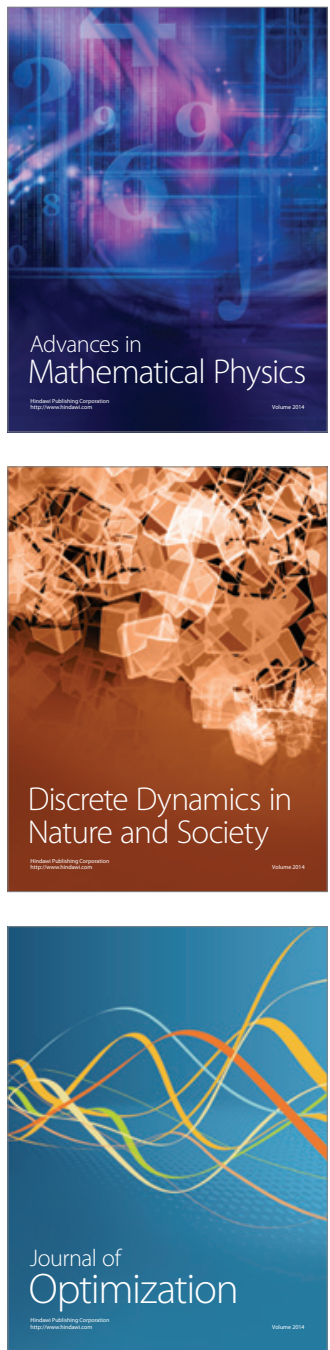